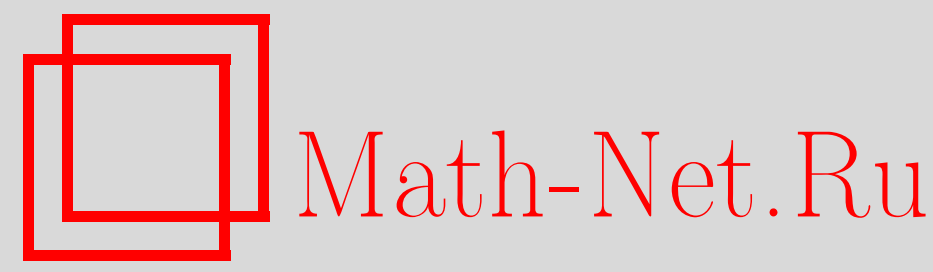

В. В. Штепин, Промежуточная ортогональная алгебра Ли $\mathfrak{b}_{n-1 / 2}$ и ее конечномерные представления, Изв. РАН. Сер. матем., 1998, том 62, выпуск 3, 201-223

DOI: https://doi.org/10.4213/im199

Использование Общероссийского математического портала Math-Net.Ru подразумевает, что вы прочитали и согласны с пользовательским соглашением

http://www . mathnet.ru/rus/agreement

Параметры загрузки:

IP : 54.224 .135 .184

26 апреля 2023 г., 15:39:00 
УДК 519.46

\section{В. В. Штепин}

\section{Промежуточная ортогональная алгебра Ли $\mathfrak{b}_{n-1 / 2}$ и ее конечномерные представления}

\footnotetext{
Предложен метод разделения кратных точек спектра в редукции $B_{n} \downarrow B_{n-1} \mathrm{c}$ помощью введения неполупростой промежуточной подалгебры. Исследуется категория модулей над этой промежуточной подалгеброй, играющих роль модулей со старшим весом.

Библиографиия: 11 наименований.
}

Одна из известных проблем спектрального анализа конечномерных представлений классических групп Ли состоит в следующем. Рассматривается конечномерное неприводимое представление $T_{m}$ комплексной простой группы Ли $G$ серии $A_{n}$, $B_{n}, C_{n}$ или $D_{n}\left(m-\right.$ старший вес представления $\left.T_{m}\right)$. Требуется описать спектр сужения представления $T_{m}$ на максимальную одноименную подгруппу $G_{0}$ меньшего ранга (соответственно $A_{n-1}, B_{n-1}, C_{n-1}$ или $D_{n-1}$ ). Решение этой проблемы хорошо известно. Оно получено Г. Вейлем [1] (для унимодулярной серии; применяя современную терминологию, Г. Вейль рассматривал алгебру Ли $\mathfrak{g l}(n, \mathbf{C}))$, И. М. Гельфандом и М. Цетлиным [2] (для ортогональных серий), Д. П. Желобенко [3] (для симплектической серии). Во всех четырех сериях спектр может содержать кратные точки.

Предположим, что спектр сужения $\left.T_{m}\right|_{G_{0}}$ не прост. Традиционный метод "освобождения от кратностей” (метод Гельфанда-Цетлина) состоит в разделении кратных точек с помощью включения между группами Ли $G_{0}$ и $G$ одной или нескольких промежуточных полупростых (или редуктивных) подгрупп $G_{1}, \ldots, G_{s-1}$ :

$$
G_{0} \subset G_{1} \subset \cdots \subset G_{s-1} \subset G_{s}=G
$$

таких, что спектр сужения каждого неприводимого $G_{i}$-подмодуля модуля $T_{m}$ на $G_{i-1}(i=1,2, \ldots, s)$ однократен. Наиболее ярким примером использования метода Гельфанда-Цетлина является построение ортогональных базисов в пространстве конечномерного неприводимого представления группы $\mathrm{GL}(n, \mathbf{C})$ с помощью цепочки вложенных подгрупп

$$
\mathbf{C}^{*} \simeq \mathrm{GL}(1, \mathbf{C}) \subset \mathrm{GL}(2, \mathbf{C}) \subset \cdots \subset \mathrm{GL}(n, \mathbf{C})
$$

и в пространстве конечномерного неприводимого представления группы $\mathrm{SO}(n, \mathbf{C})$ с помощью цепочки вложенных подгрупп

$$
\mathbf{T} \simeq \operatorname{SO}(2, \mathbf{C}) \subset \mathrm{SO}(3, \mathbf{C}) \subset \cdots \subset \mathrm{SO}(n, \mathbf{C})
$$


Однако в редукциях $A_{n} \downarrow A_{n-1}$ и $C_{n} \downarrow C_{n-1}$ метод Гельфанда-Цетлина неприменим (по причине отсутствия между $A_{n}$ и $A_{n-1}, C_{n}$ и $C_{n-1}$ промежуточных полупростых подгрупп), а в редукциях $B_{n} \downarrow B_{n-1}$ и $D_{n} \downarrow D_{n-1}$ он приводит к построению базиса в пространстве представления, не являющегося весовым.

В работах [4], [5] автором предложено обобшение метода Гельфанда-Цетлина, используюшее неполупростые промежуточные подгруппы $A_{n-1 / 2}$ (для унимодулярной серии) и $C_{n-1 / 2}$ (для симплектической серии). Показано, что “освобождения от кратностей” можно добиться не за счет разложения $T_{m}$ в прямую сумму $G_{n-1 / 2}$-модулей $\left(G_{n-1 / 2}=A_{n-1 / 2}\right.$ или $\left.G_{n-1 / 2}=C_{n-1 / 2}\right)$, а путем построения в $T_{m}$ фильтрации $G_{n-1 / 2}$-модулей. Возникающие при этом факторы фильтраций образуют категорию $\mathscr{L}$ конечномерных $G_{n-1 / 2}$-модулей, играющих роль представлений со старшим весом. В частности, для них справедливы формулы для характеров и размерностей, аналогичные формулам Г. Вейля для характеров и размерностей неприводимых представлений классических групп Ли.

Настоящая работа посвящена изучению редукции $B_{n} \downarrow B_{n-1}$ (серия $D$ будет рассмотрена отдельно). Как было отмечено, разделить кратные точки в этой редукции можно путем последовательных сужений $B_{n} \downarrow D_{n} \downarrow B_{n-1}$. Регулярное вложение $D_{n}$ в $B_{n}$ устроено просто (достаточно в стандартном представлении $B_{n}$ взять стабилизатор неизотропного вектора), но всякое вложение $B_{n-1}$ в $D_{n}$ не является регулярным, в результате чего цепочка указанных сужений приводит к построению базиса в пространстве неприводимого представления группы $B_{n}$, не являюшегося весовым. В настояшей работе разделение кратных точек спектра в редукции $B_{n} \downarrow B_{n-1}$ достигается путем введения неполупростой промежуточной подгруппы $B_{n-1 / 2}\left(B_{n-1} \subset B_{n-1 / 2} \subset B_{n}\right)$. Использование цепочки сужений $B_{n} \downarrow B_{n-1 / 2} \downarrow B_{n-1}$ в некоторых вопросах представляется предпочтительнее цепочки $B_{n} \downarrow D_{n} \downarrow B_{n-1}$. В данной работе оно позволяет явно построить весовой базис в пространстве конечномерного неприводимого представления группы $B_{n}$. Далее, при нашем подходе промежуточные строчки в таблицах Гельфанда-Цетлина, нумеруюших базисные векторы в пространстве неприводимого представления $B_{n}$, получают естественное истолкование в терминах конечномерных представлений группы Ли $B_{n-1 / 2}$. Эти конечномерные представления группы $B_{n-1 / 2}$ образуют категорию $\mathscr{L}$, элементы которой находятся во взаимно однозначном соответствии с множеством $P_{n}^{B}$ старших весов группы Ли $B_{n}$. Сравнения с сериями $A$ и $C$ позволяют предположить, что категория $\mathscr{L}$ (не вполне приводимых, вообще говоря) $B_{n-1 / 2}$-модулей играет для этой неполупростой группы Ли такую же роль, какую неприводимые представления играют для классических комплексных полупростых групп Ли. В частности, модули этой категории однозначно определяются своими старшими весами. Отметим также, что представления неполупростых групп Ли представляют самостоятельный интерес ввиду их малой изученности.

В $\S 1$ мы определим "промежуточную" группу Ли $B_{n-1 / 2}$ и ее алгебру Ли $\mathfrak{b}_{n-1 / 2}$, опишем их строение, включая разложение Леви-Мальцева.

В $\S 2$ мы введем понятия полустарших и квазистарших векторов в пространстве $V_{m}$ конечномерного неприводимого представления алгебры Ли $\mathfrak{b}_{n}$ со старшим весом $m$. Эти понятия очень удобны для описания спектров сужений представлений $V_{m}$ на $\mathfrak{b}_{n-1}$.

Далее, в $\S 3$ мы изложим обобщение традиционного метода разделения крат- 
ных точек спектра (метода Гельфанда-Цетлина), использующее неполупростую промежуточную подалгебру, и докажем основную теорему о том, что сужение $V_{m}$ на $\mathfrak{b}_{n-1}$ допускает разделение изоморфных компонент с помошью промежуточной подалгебры $\mathfrak{b}_{n-1 / 2}$. В отличие от метода Гельфанда-Цетлина разделение достигается не за счет разложения $V_{m}$ в прямую сумму $\left(\mathfrak{b}_{n-1 / 2}\right)$-модулей, а путем построения в $V_{m}$ фильтрации $\left(\mathfrak{b}_{n-1 / 2}\right)$-модулей.

Множество факторов фильтраций, разделяюших изоморфные компоненты $\left(\mathfrak{b}_{n-1}\right)$-модулей $V_{m}$, образует категорию $\mathscr{L}$, являющуюся предметом изучения в $\S 4$. Каждый такой фактормодуль является циклическим $\left(\mathfrak{b}_{n-1 / 2}\right)$-модулем, порожденным образом квазистаршего вектора $q_{\lambda, m}, \lambda \in P_{n}^{D}\left(P_{n}^{D}-\right.$ множество старших весов групшы Ли $D_{n}$ ). Мы покажем, что фактормодули, порожденные образами квазистарших векторов $q_{\lambda, m}$ и $q_{\lambda^{*}, m}$ ( $\lambda$ и $\lambda^{*}$ - зеркально сопряженные веса $P_{n}^{D}$ ), изоморфны. По этой причине элементы категории $\mathscr{L}$ (рассматриваемые с точностью до изоморфизма) находятся во взаимно однозначном соответствии не с множеством $P_{n}^{D}$, что можно было бы предположить априори, исходя из правила ветвления для ортогональных групा Ли, а с множеством $P_{n}^{B}$. Это объясняет на первый взгляд неочевидное отнесение рассматриваемой промежуточной группы Ли именно к серии $B$.

\section{§ 1. Группа Ли $B_{n-1 / 2}$ и ее алгебра Ли}

Комплексной простой группой Ли типа $B_{n}$ называют собственную ортогональную группу $\mathrm{SO}(2 n+1)$, состоящую из всех ортогональных преобразований комплексного $(2 n+1)$-мерного пространства $\mathbf{C}^{2 n+1}$, не изменяюших ориентацию. Выберем в $\mathbf{C}^{2 n+1}$ специальный базис $e_{1}, \ldots, e_{n}, e_{0}, e_{-n}, \ldots, e_{-1}$, в котором скалярное произведение определяется формой

$$
(x, y)=x_{1} y_{-1}+\cdots+x_{n} y_{-n}+x_{0} y_{0}+x_{-n} y_{n}+\cdots+x_{-1} y_{1} .
$$

В этом случае каждый элемент $g$ группы $\mathrm{SO}(2 n+1)$ удовлетворяет матричному уравнению

$$
\sigma^{-1} \cdot g \cdot \sigma=\left(g^{t}\right)^{-1}
$$

где

$$
\sigma=\left(\begin{array}{ccc}
0 & 0 & s \\
0 & 1 & 0 \\
s & 0 & 0
\end{array}\right), \quad S=\left(\begin{array}{ccccc}
0 & 0 & \ldots & 0 & 1 \\
0 & 0 & \ldots & 1 & 0 \\
\vdots & \vdots & \ddots & \vdots & \vdots \\
0 & 1 & \ldots & 0 & 0 \\
1 & 0 & \ldots & 0 & 0
\end{array}\right)
$$

"Промежуточная" подгруппа $B_{n-1 / 2}$ может быть определена как стабилизатор ненулевого изотропного вектора в пространстве стандартного представления группы Ли $\mathrm{SO}(2 n+1)$. Так как стабилизатор всегда является замкнутой подгруппой, $B_{n-1 / 2}$ - замкнутая подгруппа комплексной группы Ли, и, следовательно, сама является комплексной группой Ли.

Зафиксируем выбор вложений $\mathrm{SO}(2 n-1) \subset B_{n-1 / 2} \subset \mathrm{SO}(2 n+1)$ следуюшим образом. Подгруппа $B_{n-1 / 2}$ группы Ли $\mathrm{SO}(2 n+1)$ является стабилизатором $n$-го базисного вектора $e_{n}$ пространства $\mathbf{C}^{2 n+1}$, а $\mathrm{SO}(2 n-1)$ наряду с $e_{n}$ оставляет 
неподвижным также базисный вектор $e_{-n}$. Аналогично определяются вложения алгебр Ли $\mathfrak{s o}(2 n-1) \subset \mathfrak{b}_{n-1 / 2} \subset \mathfrak{s} o(2 n+1)$ (для "промежуточной" алгебры Ли группы $B_{n-1 / 2}$ мы применяем обозначение $\left.\mathfrak{b}_{n-1 / 2}\right)$. Более точно, алгебру Ли $\mathfrak{s o}(2 n+1)\left(\right.$ типа $\left.\mathfrak{b}_{n}\right)$ группы Ли $\mathrm{SO}(2 n+1)$ будем отождествлять с алгеброй матрищ $\left\|a_{i j}\right\| \in \mathfrak{g l}(2 n+1)$, удовлетворяюших соотношению

$$
a_{i j}=-a_{-j,-i}
$$

она порождена образуюшими

$$
X_{i j}=E_{i j}-E_{-j,-i}
$$

где $E_{i j}$ - образующие $\mathfrak{g l}(2 n+1)$ (матричные единицы).

Коммутационные соотношения в $\mathfrak{s o}(2 n+1)$ являются следствием (1) и коммутационных соотношений в $\mathfrak{g l}(2 n+1)$; они имеют вид

$$
\left[X_{i j}, X_{k l}\right]=\delta_{k j} X_{i l}-\delta_{i l} X_{k j}+\delta_{-l, j} X_{k,-i}-\delta_{k,-i} X_{-j, l} .
$$

Подалгебра $\mathfrak{b}_{n-1 / 2}$ выделяется из $\mathfrak{s o}(2 n+1)$ наложением условий $a_{i n}=0$, $a_{-n, i}=0 \quad(i=0, \pm 1, \ldots, \pm n)$, a $\mathfrak{s o}(2 n-1)$ выделяется из $\mathfrak{b}_{n-1 / 2}$ с помошью соотношений $a_{n j}=0, a_{j,-n}=0(j=0, \pm 1, \ldots, \pm n)$.

Подалгебра Картана $\mathfrak{h}_{n}$ алгебры Ли $\mathfrak{s o}(2 n+1)$ порождена образуюшими $X_{i i}$, $1 \leqslant i \leqslant n$. Пусть $R_{n}$ - система корней алгебры Ли $\mathfrak{s o}(2 n+1), P\left(R_{n}\right)-$ eе группа весов. В качестве базиса $B$ в $P\left(R_{n}\right)$ выберем элементы $\epsilon_{1}, \ldots, \epsilon_{n} \in \mathfrak{h}_{n}^{*}$, определенные равенствами $\epsilon_{i}\left(X_{j j}\right)=\delta_{i j}, 1 \leqslant i, j \leqslant n$. Тогда

$$
R_{n}=\left\{ \pm \epsilon_{i}, 1 \leqslant i \leqslant n ; \pm \epsilon_{i} \pm \epsilon_{j}, 1 \leqslant i<j \leqslant n\right\},
$$

простыми корнями системы $R_{n}$ являются

$$
\alpha_{1}=\epsilon_{1}-\epsilon_{2}, \ldots, \alpha_{n-1}=\epsilon_{n-1}-\epsilon_{n}, \alpha_{n}=\epsilon_{n} .
$$

Система корней $R_{n-1 / 2}$ алгебры Ли $\mathfrak{b}_{n-1 / 2}$ состоит из следующих корней:

$$
R_{n-1 / 2}=\left\{\alpha \in R_{n} \mid\left(\alpha, \epsilon_{n}\right) \geqslant 0\right\},
$$

где $(.,$.$) - произвольное скалярное произведение в \mathfrak{h}_{n}^{*}$, инвариантное относительно групшы Вейля. Соответственно система корней $R_{n-1}$ алгебры Ли so $(2 n-1)$ состоит из следующих корней:

$$
R_{n-1}=\left\{\alpha \in R_{n} \mid\left(\alpha, \epsilon_{n}\right)=0\right\} .
$$

Пусть $\mathfrak{s o}(2 n+1)=\mathfrak{n}_{-} \oplus \mathfrak{h}_{n} \oplus \mathfrak{m}_{+}-$разложение Картана-Вейля алгебры Ли $\mathfrak{s o}(2 n+1)$, согласованное с базисом $B, \mathfrak{b}^{ \pm}=\mathfrak{h}_{n} \oplus \mathfrak{n}_{ \pm}$- соответствующие борелевские подалгебры. Положим $\mathfrak{m}_{ \pm}=\mathfrak{n}_{ \pm} \cap \mathfrak{b}_{n-1 / 2}$. Мы имеем тогда следующее разложение, аналогичное разложению Картана-Вейля:

$$
\mathfrak{b}_{n-1 / 2}=\mathfrak{m}_{-} \oplus \mathfrak{h}_{n-1} \oplus \mathfrak{m}_{+} .
$$

Алгебры Ли $\mathfrak{b}_{n-1 / 2}^{-}=\mathfrak{h}_{n-1} \oplus \mathfrak{m}_{-}$и $\mathfrak{b}_{n-1 / 2}^{+}=\mathfrak{h}_{n-1} \oplus \mathfrak{m}_{+}$являются аналогами борелевских подалгебр в $\mathfrak{b}_{n-1 / 2}$.

Докажем следуюшее простое предложение, которое дает разложение Леви-Мальцева групшы Ли $B_{n-1 / 2}$.

ПРЕДЛОЖенИЕ 1.1. Группа Ли $B_{n-1 / 2}$ не редуктивна. Она изоморфна

$$
B_{n-1 / 2} \simeq \mathrm{SO}(2 n-1) \ltimes \mathbf{C}^{2 n-1},
$$

əде $\mathbf{C}^{2 n-1}$ - аддитивная группа Ли, $\ltimes$ - полупрямое произведение групп Ли. 
ДокАЗАТЕЛЬСТво. Пусть $x_{i}(t)$ (соответственно $\left.y_{i}(t)\right), 1 \leqslant i \leqslant n-1,-$ однопараметрическая подгруппа в $B_{n-1 / 2}$, соответствуюшая образующему $X_{n i}$ (соответственно $\left.X_{i,-n}\right)$ алгебры Ли $\mathfrak{b}_{n-1 / 2}$. Пусть также $z(t)$ - однопараметрическая подгруппа в $B_{n-1 / 2}$, соответствуюшая образуюшему $X_{n 0}$ алгебры Ли $\mathfrak{b}_{n-1 / 2}$. Однопараметрические подгрупшы $x_{i}(t), y_{i}(t), 1 \leqslant i \leqslant n-1$, и $z(t)$ порождают мультипликативную абелеву группу Ли $H$ (абелевость вытекает из того, что образуюшие $X_{n i}, X_{j,-n}, X_{n 0}, 1 \leqslant i, j \leqslant n-1$, попарно коммутируют в $\left.\mathfrak{s o}(2 n+1)\right)$, изоморфную аддитивной группе $\mathbf{C}^{2 n-1}$. Далее, группа $\mathrm{SO}(2 n-1) \ltimes H$ есть по определению множество упорядоченных пар $(s, h), s \in \mathrm{SO}(2 n-1), h \in H$, с групшовым умножением $\left(s_{1}, h_{1}\right) \cdot\left(s_{2}, h_{2}\right)=\left(s_{1} s_{2}, h_{1} s_{1} h_{2} s_{1}^{-1}\right)$. Определим аналитическое отображение $\varphi: \mathrm{SO}(2 n-1) \ltimes H \rightarrow B_{n-1 / 2}$ равенством $\varphi(s, h)=h s$. Тогда $\varphi$ осушествляет искомый изоморфизм (2).

СлЕДСТВИЕ 1.1. Алгебра Ли $\mathfrak{b}_{n-1 / 2}$ имеет нетривиальный радикал $r$, изоморфный коммутативной алгебре Ли размерности $2 n-1$, при этом

$$
\mathfrak{b}_{n-1 / 2} \simeq \mathfrak{s o}(2 n-1) \dot{+} r
$$

где $\dot{+}-$ полупрямая сумма алгебр Ли.

Алгебра Ли $\mathfrak{b}_{n}$ обладает каноническим инволютивным автоморфизмом $\Theta$, переставляюшим положительные и отрицательные корневые векторы $\mathfrak{b}_{n}$. Автоморфизм $\Theta$ можно определить следующим образом: пусть $R_{n}^{+}$- множество положительных корней алгебры Ли $\mathfrak{b}_{n}$ относительно базиса $B$, тогда для любого $\alpha \in R_{n}^{+}$

$$
\Theta\left(X_{\alpha}\right)=-X_{-\alpha}, \quad \Theta\left(X_{-\alpha}\right)=-X_{\alpha}
$$

а на подалгебре Картана $\mathfrak{h}_{n}$ алгебры Ли $\mathfrak{b}_{n}$ автоморфизм $\Theta$ действует как "минус-тождественный" оператор. Сразу отметим, что этот автоморфизм $\Theta$ не является автоморфизмом $\mathfrak{b}_{n-1 / 2}$ (так как не сохраняет эту подалгебру Ли).

С другой стороны, канонический инволютивный автоморфизм алгебры Ли $\mathfrak{b}_{n-1}$ может быть двумя способами продолжен до инволютивного автоморфизма алгебры Ли $\mathfrak{b}_{n-1 / 2}$. Обозначим эти продолжения через $\Theta_{+}$и $\Theta_{-}$. Поскольку $\Theta_{+}$и $\Theta_{-}$ совпадают на $\mathfrak{b}_{n-1}$, различить их можно лиш по их действию на корневые векторы $\mathfrak{b}_{n-1 / 2}$, дополнительные к $\mathfrak{b}_{n-1}$. Среди этих корневых векторов в точности один является собственным для автоморфизмов $\Theta_{+}$и $\Theta_{-}$, это вектор $X_{n 0}$, отвечаюший последнему простому корню $\alpha_{n}$ алгебры Ли $\mathfrak{b}_{n}$. Автоморфизмы $\Theta_{+} \Theta_{-}$ отличаются своими собственными значениями на векторе $X_{n 0}$ :

$$
\Theta_{ \pm} X_{n 0}= \pm X_{n 0}
$$

Как будет показано в $\S 3$, корневой вектор $X_{n 0}$ имеет исключительное значение не только для различения автоморфизмов $\Theta_{+}$и $\Theta_{-}$алгебры Ли $\mathfrak{b}_{n-1 / 2}$, но и для различения изоморфных зеркально сопряженных $\left(\mathfrak{b}_{n-1 / 2}\right)$-факторов, что является составной частью нашего метода (см. определение 3.3 ). 


\section{§ 2. Полустаршие и квазистаршие векторы}

Пусть $P_{n}^{B}$ - множество старших весов алгебры Ли $\mathfrak{s o}(2 n+1), P_{n}^{B} \subset \frac{1}{2} P\left(R_{n}\right)$. Хорошо известно (см., например, [6]), что в определенном вьше базисе $\epsilon_{1}, \ldots, \epsilon_{n}$ эти веса задаются следуюшими целочисленными или полуцелочисленными векторами:

$$
P_{n}^{B}=\left\{\left(m_{1}, \ldots, m_{n}\right) ; m_{1} \geqslant m_{2} \geqslant \cdots \geqslant m_{n} \geqslant 0, m_{i} \in \mathbf{Z} \text { или } m_{i} \in \mathbf{Z}+\frac{1}{2}\right\} .
$$

Старшие векторы с полуцелыми компонентами необходимы для описания двузначных представлений группы Ли $\mathrm{SO}(2 n+1)$. Обозначим через $V_{m}$ конечномерный неприводимый $\mathfrak{s o}(2 n+1)$-модуль со старшим весом $m \in P_{n}^{B}$. Известно [7], что $V_{m}$ обладает единственным с точностью до постоянного множителя контравариантным скалярным произведением $\langle.$, . $\rangle$. (Контравариантность означает, что операторы представления, соответствуюшие образуюшим $X_{i j}$ и $X_{j i}$, эрмитово сопряжены относительно $\langle.,$.$\rangle .) Всюду ниже под ортогональностью векторов в V_{m}$ мы будем подразумевать ортогональность относительно этого скалярного произведения. Определяемые ниже понятия полустарших и квазистарших векторов очень удобны в вопросах спектрального анализа сужений $V_{m}$ на $\mathfrak{s o}(2 n-1)$ и $\mathfrak{b}_{n-1 / 2}$ и будут использованы нами в $\S 3$.

ОПРЕДЕЛЕНИЕ 2.1. Вектор $v \in V_{m}$, собственньй относительно $\mathfrak{h}_{n}$ и старший относительно $\mathfrak{s o}(2 n-1)$, называется полустариим.

ОПРЕДЕЛЕНИЕ 2.2 (ср. [4], [8]). Полустарший вектор $v \in V_{m}$, удовлетворяющий соотношениям $X_{i n} v=0, i=1,2, \ldots, n-1$, называется квазистариим. Обозначим через $V_{m}^{+}$(соответственно $V_{m}^{++}$) линейную оболочку множества полустарших (соответственно квазистарших) векторов. Подпространство $V_{m}^{+}$мы будем называть пространством полустариих векторов, соответственно $V_{m}^{++}$ пространством квазистариих векторов.

Сформулируем без доказательства два простых предложения, описываюших свойства пространства полустарших векторов.

ПРЕДЛОЖЕНИЕ 2.1. Стариий вектор $v$ любого неприводимого $\mathfrak{s o}(2 n-1)$ подмодуля модуля $V_{m}$ принадлежит $V_{m}^{+}$.

ПрЕДЛОЖЕНИЕ 2.2. а) Пусть $\left.V_{m}\right|_{\mathfrak{s o}(2 n-1)}=\bigoplus_{i=1}^{k} V_{i}$ - некоторое разложение $V_{m}$ в прямую сумму неприводимых sо $(2 n-1)$-подмодулей. Тогда старшие векторь модулей $V_{i}, \quad 1 \leqslant i \leqslant k$, образуют базис $V_{m}^{+}$.

б) Обратно, пусть $v_{1}, \ldots, v_{k}$ образуют базис $V_{m}^{+}$, состоящий из полустарших векторов. Пусть $V_{i}-\mathfrak{s o}(2 n-1)$-подмодуль $V_{m}$, порожденный вектором $v_{i}, 1 \leqslant i \leqslant k$. Тогда модули $V_{i}, 1 \leqslant i \leqslant k$, неприводимы $u$

$$
\left.V_{m}\right|_{\mathfrak{s} o(2 n-1)}=\bigoplus_{i=1}^{k} V_{i} .
$$

Предложения 2.1 и 2.2 доказываются стандартными методами (см., например, $[9],[10])$. 
Для более наглядного описания пространств $V_{m}^{+}$и $V_{m}^{++}$мы воспользуемся конструкцией Желобенко [3], реализующей любое конечномерноенеприводимое представление $T_{m}$ классической комплексной группы Ли $G$ в пространстве многочленов на максимальной унипотентной подгруппе $Z_{+}$.

Пусть $Z_{-}$(соответственно $\left.Z_{+}\right)$- подгруппа $\mathrm{SO}(2 n+1)$, состояшая из нижнетреугольных (соответственно верхнетреугольных) матриц с единицами на главной диагонали, $H$ - подгруппа диагональных матриц. Тогда $\mathrm{SO}(2 n+1)=\overline{Z_{-} H Z_{+}}$ - разложение Гаусса и всякий регулярный элемент $g \in \mathrm{SO}(2 n+1)$ однозначно представляется в виде $g=\zeta \delta z$, где $\zeta \in Z_{-}, \delta \in H, z \in Z_{+}$. Напомним, что матрица $g \in \mathrm{SO}(2 n+1)$ называется регулярной, если все ее главные миноры, т.е. миноры, расположенные на пересечении первых $k$ строк и первых $k$ столбцов, $k=1, \ldots, 2 n+1$, отличны от нуля. Независимыми параметрами в подгруппе $Z_{+}$ являются матричные элементы, стояшие выше второй диагонали (иначе говоря, это элементы $z_{p q}$ такие, что $0<p<|q|$, а также элементы $z_{p 0}, 1 \leqslant p \leqslant n$ ). Согласно [3] всякое конечномерное неприводимое представление $\mathrm{SO}(2 n+1)$ может быть реализовано в $\mathbf{C}\left[z_{p q}\right]$. Пространство неприводимого представления $T_{m}$ группы Ли $\mathrm{SO}(2 n+1)$ со старшим весом $m \in P_{n}^{B}$ является пространством решений системы дифференциальных уравнений (индикаторной системы)

$$
\left\{\begin{array}{l}
D_{1}^{r_{1}+1} f(z)=0 \\
\cdots \cdots \cdots \cdots \cdots \\
D_{n}^{r_{n}+1} f(z)=0
\end{array}\right.
$$

где $f(z) \in \mathbf{C}\left[z_{p q}\right], r_{i}=m_{i}-m_{i+1}, i=1, \ldots, n-1, r_{n}=2 m_{n}$, а $D_{1}, \ldots, D_{n}$ - так называемые "главные сдвиги", т.е. инфинитезимальные операторы левых сдвигов на подгруппе $Z_{+}$, соответствуюших простым корням $\mathrm{SO}(2 n+1)$. Это пространство мы обозначим через $V_{m}$, как и соответствуюший $\mathfrak{s o}(2 n+1)$-модуль. Операторы $D_{1}, \ldots, D_{n}$ могут быть вьписаны явно:

$$
\begin{aligned}
& D_{1}=\frac{\partial}{\partial z_{12}}+z_{23} \frac{\partial}{\partial z_{13}}+\cdots+z_{2 n} \frac{\partial}{\partial z_{1 n}}+z_{20} \frac{\partial}{\partial z_{10}}+z_{2,-n} \frac{\partial}{\partial z_{1,-n}}+\cdots \\
& \cdots+z_{2,-3} \frac{\partial}{\partial z_{1,-3}}-\left(\frac{z_{20}^{2}}{2}+z_{23} z_{2,-3}+\cdots+z_{2 n} z_{2,-n}\right) \frac{\partial}{\partial z_{1,-2}}, \\
& D_{2}=\frac{\partial}{\partial z_{23}}+z_{34} \frac{\partial}{\partial z_{24}}+\cdots+z_{3 n} \frac{\partial}{\partial z_{2 n}}+z_{30} \frac{\partial}{\partial z_{20}}+z_{3,-n} \frac{\partial}{\partial z_{2,-n}}+\cdots \\
& \cdots+z_{3,-4} \frac{\partial}{\partial z_{2,-4}}-\left(\frac{z_{30}^{2}}{2}+z_{34} z_{3,-4}+\cdots+z_{3 n} z_{3,-n}\right) \frac{\partial}{\partial z_{2,-3}}, \\
& D_{n-1}=\frac{\partial}{\partial z_{n-1, n}}+z_{n 0} \frac{\partial}{\partial z_{n-1,0}}-\frac{z_{n 0}^{2}}{2} \frac{\partial}{\partial z_{n-1,-n}}, \quad D_{n}=\frac{\partial}{\partial z_{n 0}} .
\end{aligned}
$$

Далее, пусть $\mathfrak{n}_{ \pm}-$алгебра Ли группы $Z_{ \pm}$и $_{ \pm}^{0}=\mathfrak{n}_{ \pm} \cap \mathfrak{s o}(2 n-1)$. Обозначим через $V^{+}$подпространство всех многочленов в $\mathbf{C}\left[z_{p q}\right]$, аннулируемых подалгеброй $\mathfrak{n}_{+}^{0}$. Легко видеть, что

$$
V^{+}=\mathbf{C}\left[z_{1 n}, \ldots, z_{n-1, n}, z_{1,-n}, \ldots, z_{n-1,-n}, z_{n 0}\right],
$$


и теперь $V_{m}^{+}=V^{+} \cap V_{m}$.

Далее, пусть $V^{++}-$подпространство всех многочленов в $V^{+}$, аннулируемых операторами $X_{i n}, i=1, \ldots, n-1$. В используемой реализации инфинитезимальные операторы $X_{\text {in }}$ имеют следуюший вид:

$$
\begin{aligned}
& X_{1 n}= \frac{\partial}{\partial z_{1 n}}, \\
& X_{2 n}= \frac{\partial}{\partial z_{2 n}}+z_{12} \frac{\partial}{\partial z_{1 n}}-z_{1,-n} \frac{\partial}{\partial z_{1,-2}}, \\
& \ldots \ldots \ldots \ldots \ldots \ldots \ldots \ldots \ldots \ldots \ldots \ldots \ldots \ldots \\
& X_{n-2, n}= \frac{\partial}{\partial z_{n-2, n}}+z_{n-3, n-2} \frac{\partial}{\partial z_{n-3, n}}+\cdots+z_{1, n-2} \frac{\partial}{\partial z_{1 n}} \\
&-z_{n-3,-n} \frac{\partial}{\partial z_{n-3,-(n-2)}}-\cdots-z_{1,-n} \frac{\partial}{\partial z_{1,-(n-2)}}, \\
& X_{n-1, n}= \frac{\partial}{\partial z_{n-1, n}}+z_{n-2, n-1} \frac{\partial}{\partial z_{n-2, n}}+\cdots+z_{1, n-1} \frac{\partial}{\partial z_{1 n}} \\
&-z_{n-2,-n} \frac{\partial}{\partial z_{n-2,-(n-1)}}-\cdots-z_{1,-n} \frac{\partial}{\partial z_{1,-(n-1)}} .
\end{aligned}
$$

Отсюда имеем $V^{++}=\mathbf{C}\left[z_{1,-n}, \ldots, z_{n-1,-n}, z_{n 0}\right]$ и $V_{m}^{++}=V^{++} \cap V_{m}$.

ПРЕДЛОЖЕНИЕ 2.3. Система квазистариих векторов

$$
\left\{z_{1,-n}^{k_{1}} \ldots z_{n-1,-n}^{k_{n-1}} z_{n 0}^{k_{n}} ; 0 \leqslant k_{i} \leqslant r_{i}, k_{i} \in \mathbf{Z}, i=1, \ldots, n\right\}
$$

образует ортогональный базис пространства квазистариих векторов $V_{m}^{++}$.

ДокАЗАТЕльство. На подпространстве $V^{++}$“главные сдвиги” имеют следуюший вид:

$$
\begin{aligned}
D_{1}=z_{2,-n} \frac{\partial}{\partial z_{1,-n}}, \quad D_{2} & =z_{3,-n} \frac{\partial}{\partial z_{2,-n}}, \quad \ldots \\
\ldots, \quad D_{n-1} & =-\frac{z_{n 0}^{2}}{2} \frac{\partial}{\partial z_{n-1,-n}}, \quad D_{n}=\frac{\partial}{\partial z_{n 0}} .
\end{aligned}
$$

Базис в пространстве решений индикаторной системы (3), принадлежаших $V^{++}$, т.е. базис в пространстве $V_{m}^{++}$, образуют мономы вида $z_{1,-n}^{k_{1}} z_{2,-n}^{k_{2}} \ldots z_{n-1,-n}^{k_{n-1}} z_{n 0}^{k_{n}}$, где $k_{i} \in \mathbf{Z}, 0 \leqslant k_{i} \leqslant r_{i}, i=1, \ldots, n$. Легко видеть, что моном $z_{1,-n}^{k_{1}} \ldots z_{n-1,-n}^{k_{n-1}} z_{n 0}^{k_{n}}$ - весовой вектор $V_{m}$. Его вес в базисе $\epsilon_{1}, \ldots, \epsilon_{n}$ таков:

$$
\left(m_{1}-k_{1}, m_{2}-k_{2}, \ldots, m_{n-1}-k_{n-1}, m_{n}-\sum_{i=1}^{n} k_{i}\right) .
$$

Но собственные векторы эрмитовых операторов, имеющие различные собственные значения, взаимно ортогональны.

Можно показать, что в пространстве полустарших векторов $V_{m}^{+}$не существует, вообще говоря, ортогональный базис, состоящий из мономов от независимых 
параметров $z_{p q}$ группы $Z_{+}$. Тем не менее, вычисляя размерности весовых подпространств пространства $V_{m}^{+}$, можно получить правило ветвления для редукции $B_{n} \downarrow B_{n-1}$. Это правило ветвления впервые было получено Гельфандом и Цетлиным в [2]. Их результат:

$$
\left.V_{\left(m_{1}, \ldots, m_{n}\right)}\right|_{B_{n-1}}=\bigoplus V_{\left(m_{1}^{\prime \prime}, \ldots, m_{n-1}^{\prime \prime}\right)},
$$

где прямая сумма берется по всем наборам векторов $m^{\prime \prime}=\left(m_{1}^{\prime \prime}, \ldots, m_{n-1}^{\prime \prime}\right), m^{\prime}=$ $\left(m_{1}^{\prime}, \ldots, m_{n}^{\prime}\right)$, одновременно целочисленных (если вектор $m=\left(m_{1}, \ldots, m_{n}\right)$ целочисленный) или полуцелочисленных (если вектор $m=\left(m_{1}, \ldots, m_{n}\right)$ полуцелочисленньй), удовлетворяющим системе неравенств

$$
\begin{array}{r}
m_{1} \geqslant m_{1}^{\prime} \geqslant m_{2} \geqslant \cdots \geqslant m_{n-1} \geqslant m_{n-1}^{\prime} \geqslant m_{n} \geqslant\left|m_{n}^{\prime}\right|, \\
m_{1}^{\prime} \geqslant m_{1}^{\prime \prime} \geqslant m_{2}^{\prime} \geqslant \cdots \geqslant m_{n-1}^{\prime} \geqslant m_{n-1}^{\prime \prime} \geqslant\left|m_{n}^{\prime}\right| .
\end{array}
$$

Правило ветвления (4) может быть переписано в следующем, более компактном виде, использующем условия подчинения весов.

Пусть $P_{n}^{D}$ - множество старших весов алгебры Ли $s o(2 n)$ (типа $D_{n}$ ). Хорошо

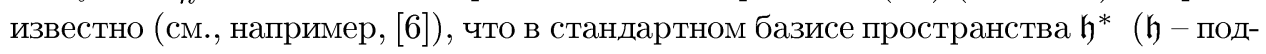
алгебра Картана алгебры Ли so $(2 n))$ эти веса задаются следуюшими целочисленными или полуцелочисленными векторами:

$$
\begin{aligned}
P_{n}^{D}=\left\{\left(m_{1}, \ldots, m_{n}\right) ; m_{1} \geqslant m_{2} \geqslant \cdots \geqslant m_{n-1} \geqslant\left|m_{n}\right|, m_{i} \in \mathbf{Z}\right. \\
\text { или } \left.m_{i} \in \mathbf{Z}+\frac{1}{2}\right\} .
\end{aligned}
$$

Отметим попутно, что последняя компонента старшего веса $m \in P_{n}^{D}$ может оказаться отрицательной (в отличие от $m \in P_{n}^{B}$ ). Будем говорить, что вес $l \in P_{i}^{D}$ подчинен весу $m \in P_{i}^{B}$, если их компоненты удовлетворяют системе неравенств $m_{1} \geqslant l_{1} \geqslant m_{2} \geqslant \cdots \geqslant m_{i-1} \geqslant l_{i-1} \geqslant m_{i} \geqslant\left|l_{i}\right|$. Запись $l \rightarrow m$ для весов $l \in P_{i}^{D}$, $m \in P_{i}^{B}$ будет означать, что $l$ подчинен $m$. Далее, будем говорить, что вес $t \in P_{j}^{B}$ подчинен весу $l \in P_{j+1}^{D}$, если их компоненты удовлетворяют системе неравенств $l_{1} \geqslant t_{1} \geqslant l_{2} \geqslant \ldots \geqslant l_{j} \geqslant t_{j} \geqslant\left|l_{j+1}\right|$. И в этом случае запись $t \rightarrow l$ для весов $t \in P_{j}^{B}, l \in P_{j+1}^{D}$ будет означать, что $t$ подчинен $l$.

Теперь правило ветвления (4) для редукции $\mathfrak{s o}(2 n+1) \downarrow \mathfrak{s o}(2 n-1)$ может быть записано более компактно. Пусть $V_{m}$ - конечномерный неприводимый so $(2 n+1)$-модуль со старшим весом $m \in P_{n}^{B}$. Его сужение на $\mathfrak{s o}(2 n-1)$ вполне приводимо и имеет следующий спектр:

$$
\left.V_{m}\right|_{\mathfrak{s} o(2 n-1)}=\bigoplus_{l \rightarrow m}\left(\bigoplus_{t \rightarrow l} V_{t}\right), \quad t \in P_{n-1}^{B}, \quad l \in P_{n}^{D},
$$

причем в обеих суммах веса $l$ и $t$, по которьм идет суммирование, одновременно целочисленные, если вектор $m$ целочислен, либо полуцелочисленные, если вектор $m$ имеет полуцелые компоненты. 
ЗАмечАниЕ. Правило ветвления, записанное в виде (5), наводит на мысль, что пространство $\underset{t \rightarrow l}{\oplus} V_{t}$ (суммирование по $t \in P_{n-1}^{B} ; l \in P_{n}^{D}$ фиксирован) может быть наделено структурой не только $\mathfrak{s o}(2 n-1)$-модуля, но также структурой модуля над некоторой промежуточной между $\mathfrak{s o}(2 n-1)$ и $\mathfrak{s o}(2 n+1)$ алгеброй Ли.

Как было отмечено во введении, в качестве такой промежуточной алгебры Ли естественно взять $\mathfrak{s o}(2 n)$; тогда $\underset{t \rightarrow l}{\oplus} V_{t}$ наделяется структурой $\mathfrak{s o}(2 n)$-модуля со старшим весом $l \in P_{n}^{D}$. Однако такой подход имеет единственный недостаток (который мы собираемся устранить в $\S 3,4)$, а именно цепочка последовательных сужений $\mathfrak{s o}(2 n+1) \downarrow \mathfrak{s} o(2 n), \mathfrak{s} o(2 n) \downarrow \mathfrak{s} o(2 n-1), \ldots$ приводит к построению базиса в пространстве $V_{m}$, не являющегося весовым. Ниже мы получим другое истолкование “промежуточных строчек" $l \in P_{n}^{D}$ в правиле ветвления (5) с точки зрения теории представлений неполупростых алгебр Ли (отметим, что наш подход представляется более универвальным, поскольку он применим также в редукциях унимодулярных и симплектических алгебр Ли [4]).

\section{§ 3. Разделение изоморфных компонент}

Пусть $\mathfrak{g}, \mathfrak{g}_{0}$ - полупростые комплексные алгебры Ли, причем $\mathfrak{g}_{0}$ регулярно вложена в $\mathfrak{g}$. Пусть $V$ - неприводимый конечномерный $\mathfrak{g}$-модуль такой, что спектр сужения $\left.V\right|_{\mathfrak{g}_{0}}$ не прост. Что следует понимать под разделением изоморфинх компонент $\mathfrak{g}_{0}$-модуля $V ?$ На этот вопрос можно ответить по-разному.

Традиционный метод (метод Гельфанда-Цетлина) предусматривает использование одной или нескольких промежуточных (между $\mathfrak{g}_{0}$ и $\mathfrak{g}$ ) полупростых подалгебр. Более точно, ищется цепочка регулярно вложенных полупростых подалгебр $\mathfrak{g}_{1}, \ldots, \mathfrak{g}_{k-1}, \mathfrak{g}_{0} \subset \mathfrak{g}_{1} \subset \cdots \subset \mathfrak{g}_{k-1} \subset \mathfrak{g}_{k}=\mathfrak{g}$ таких, что сужение каждого неприводимого $\mathfrak{g}_{i}$-подмодуля модуля $V$ на $\mathfrak{g}_{i-1}(i=1,2, \ldots, k)$ имеет простой спектр. Отметим сразу, что этот метод применим не всегда. Может существовать неприводимый $\mathfrak{g}_{i}$-модуль $W, W \subseteq V$, такой, что спектр сужения $\left.W\right|_{\mathfrak{g}_{i-1}}$ не прост, а между $\mathfrak{g}_{i-1}$ и $\mathfrak{g}_{i}$ нет никакой промежуточной полупростой подалгебры. Как раз такое положение имеет место в редукциях $\mathfrak{g}_{n} \downarrow \mathfrak{g}_{n-1}$, где $\mathfrak{g}_{n}-$ унимодулярная $(\mathfrak{s l}(n+1))$ или симплектическая $(\mathfrak{s} p(2 n))$ алгебра Ли.

В работах [4], [5] было предложено обобщение традиционного метода, допускающее использование промежуточной (между $\mathfrak{g}_{i-1}$ и $\mathfrak{g}_{i}$ ) подалгебры, не обязательно полупростой. Точная формулировка этого метода (см. [4]) заключена в следующих двух определениях.

ОПРЕДЕЛЕНИЕ 3.1. Для всякой фильтрации

$$
f=\left\{V_{0}, V_{1}, \ldots, V_{s} ; V_{i-1} \subset V_{i}, i=1,2, \ldots, s\right\}
$$

$\mathfrak{g}$-модулей, $\mathfrak{g}$ - произвольная алгебра Ли, будем называть фактормодули $V_{i} / V_{i-1}$ факторами фильтрации $f$. Фильтрацию, у которой никакие два фактора не изоморфны, будем называть фильтрацией без кратностей. Если g-модуль $V$ наделен скалярным произведением (., . ), то прообразом фактора $V_{i} / V_{i-1}$ в $V$ будем называть ортогональное дополнение $\mathfrak{g}$-модуля $V_{i-1}$ в $V_{i}$ относительно $(.$, . ). 
ОПРЕДЕЛЕНИЕ 3.2. Пусть, как и вьше, $W$-неприводимый $\mathfrak{g}_{i}$-модуль такой, что спектр $\left.W\right|_{\mathfrak{g}_{i-1}}$ не прост. Будем говорить, что $\left(\mathfrak{g}_{i-1}\right)$-модуль $W$ допускает разделение изоморфных компонент с помощью промежуточной подалгебры $\mathfrak{g}_{i-1 / 2}\left(\mathfrak{g}_{i-1} \subset\right.$ $\left.\mathfrak{g}_{i-1 / 2} \subset \mathfrak{g}_{i}\right)$, если сушествует фильтрация $f=\left\{W_{0}, W_{1}, \ldots, W_{s} ; W_{i-1} \subset W_{i}\right.$, $\left.i=1,2, \ldots, s, W_{0}=\{0\}, W_{s}=W\right\}\left(\mathfrak{g}_{i-1 / 2}\right)$-подмодулей в $W$ такая, что:

a) $f$ - фильтрация без кратностей;

б) сужения факторов фильтрации $f$ на $\mathfrak{g}_{i-1}$ имеют простой спектр.

Такой подход позволил разделить изоморфные компоненты в редукциях $\mathfrak{g}_{n} \downarrow$ $\mathfrak{g}_{n-1}$, где $\mathfrak{g}_{n}$ - алгебра Ли типа $A_{n}$ или $C_{n}$. Естественно было ожидать, что он "сработаег" и в случае ортогональных алгебр Ли. При ближайшем рассмотрении оказалось, что определение 3.2 не годится для ортогонального случая. Как видно из правила ветвления (5), в ортогональном случае встречаются зеркально-сопряженные промежуточные веса

$$
l=\left(l_{1}, \ldots, l_{n-1}, l_{n}\right) \in P_{n}^{D} \text { и } l^{*}=\left(l_{1}, \ldots, l_{n-1},-l_{n}\right) \in P_{n}^{D} .
$$

Соответствующие этим весам факторы фильтраций $f$ (всюду ниже называемые зеркально-сопряженныцм факторами) изоморфны не только как $\left(\mathfrak{b}_{n-1}\right)$-модули (что сразу вытекает из правила ветвления (5)), но и как $\left(\mathfrak{b}_{n-1 / 2}\right)$-модули (что совсем не очевидно). Следуюшее ниже определение 3.3 разделения изоморфных компонент модифицирует определение 3.2 , учитьвая, что прообразы зеркально-сопряженных $\left(\mathfrak{b}_{n-1 / 2}\right)$-факторов в $V_{m}$ различаются по их степени нильпотентности относительно оператора $X_{n 0}$.

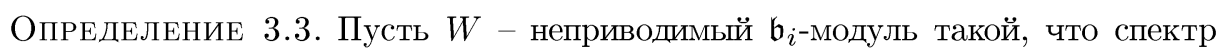
$\left.W\right|_{\mathfrak{b}_{i-1}}$ не прост. Будем говорить, что $\left(\mathfrak{b}_{i-1}\right)$-модуль $W$ допускает разделение изоморфных компонент с помошью промежуточной подалгебры $\mathfrak{b}_{i-1 / 2}\left(\mathfrak{b}_{i-1} \subset\right.$ $\left.\mathfrak{b}_{i-1 / 2} \subset \mathfrak{b}_{i}\right)$, если сушествует фильтрация

$$
f=\left\{W_{0}, W_{1}, \ldots, W_{s} ; W_{i-1} \subset W_{i}, i=1, \ldots, s, W_{0}=\{0\}, W_{s}=W\right\}
$$

$\left(\mathfrak{b}_{i-1 / 2}\right)$-подмодулей в $W$ такая, что:

а) всякие два фактора $f$ либо не изоморфны, либо, в случае зеркально-сопряженных факторов, их прообразы в $W$ имеют разную степень нильпотентности относительно корневого вектора $X_{i 0}$ (отвечающего последнему простому корню $\alpha_{i}$ алгебры Ли $\left.\mathfrak{b}_{i}\right)$;

б) сужения факторов фильтрации $f$ на $\mathfrak{b}_{i-1}$ имеют простой спектр.

Теперь если условия определения 3.3 вьполняются, то изоморфные неприводимые компоненты $\left(\mathfrak{b}_{i-1}\right)$-модуля $W$ можно различать, относя их либо к неизомор фным факторам фильтрации $f$, либо (в случае зеркально-сопряженных факторов) по их степени нильпотентности относительно оператора $X_{i 0}$.

Сформулируем основной результат данной работы.

Теорема 3.1. Пусть $V_{m}$ - конечномерный неприводимый $\mathfrak{b}_{n}$-модуль со стариим весом $m \in P_{n}^{B}$. Тогда сужение $V_{m}$ на $\mathfrak{b}_{n-1}$ допускает разделение изоморфных компонент с помощью промежуточной подалгебры $\mathfrak{b}_{n-1 / 2}$.

Доказательство теоремы 1 заключается в явном построении фильтрации $f$ $\left(\mathfrak{b}_{n-1 / 2}\right)$-подмодулей в $V_{m}$, удовлетворяющей условиям определения 3.3 . В этом 
построении существенную роль будут играть определенные в $§ 2$ квазистаршие векторы.

Введем во множестве квазистарших векторов $\mathfrak{b}_{n}$-модуля $V_{m}$ (рассматриваемых с точностью до пропорциональности) отношение линейного порядка:

$$
z_{1,-n}^{k_{1}} z_{2,-n}^{k_{2}} \ldots z_{n-1,-n}^{k_{n-1}} z_{n 0}^{k_{n}}<z_{1,-n}^{p_{1}} z_{2,-n}^{p_{2}} \ldots z_{n-1,-n}^{p_{n-1}} z_{n 0}^{p_{n}}
$$

если $\left(k_{1}, k_{2}, \ldots, k_{n-1}, k_{n}\right)<\left(p_{1}, p_{2}, \ldots, p_{n-1}, p_{n}\right)$ относительно лексикографического порядка. Перенумеруем квазистаршие векторы в соответствии с этим линейным порядком: $q_{1}=1, q_{2}=z_{n 0}, \ldots, q_{N}=z_{1,-n}^{r_{1}} \ldots z_{n-1,-n}^{r_{n-1}} z_{n 0}^{r_{n}}$. Далее, обозначим через $\mathscr{D}_{i}, i=1, \ldots, N,\left(\mathfrak{b}_{n-1 / 2}\right)$-подмодуль $V_{m}$, порожденный векторами $q_{1}, \ldots, q_{i}$. Пусть также $\mathscr{D}_{0}=0$. По построению полученные $\left(\mathfrak{b}_{n-1 / 2}\right)$-модули образуют фильтрацию: $\mathscr{D}_{i-1} \subset \mathscr{D}_{i}, i=1,2, \ldots, N$. Покажем, что $\mathscr{D}_{N}=V_{m}$. Поскольку всякий ненулевой вектор простого модуля над полупростой алгеброй Ли является циклическим, последнее равенство вытекает из $\mathfrak{b}_{n-1 / 2} q_{N}=\mathfrak{b}_{n} q_{N}$. Но $\mathfrak{b}_{n-1 / 2}$ может быть дополнена до $\mathfrak{b}_{n}$ подалгеброй

$$
\left\langle X_{1 n}, \ldots, X_{n n}, X_{-n, 1}, \ldots, X_{-n, n-1}, X_{0 n}\right\rangle
$$

Сразу заметим, что образующие $X_{1 n}, \ldots, X_{n-1, n}$ аннулируют $q_{N}$ по определению квазистаршего вектора. Далее, $X_{n n}$ сохраняет одномерное подпространство, порожденное $q_{N}$, поскольку $X_{n n} \in \mathfrak{h}_{n}$, а $q_{N}$ собственный относительно $\mathfrak{h}_{n}$. Покажем, что образующий $X_{-n, i}, 1 \leqslant i \leqslant n-1$, также аннулирует $q_{N}$. Действительно, $X_{-n i} q_{N}$ является весовым с компонентами веса в базисе $\epsilon_{1}, \ldots, \epsilon_{n}:\left(m_{2}, m_{3}, \ldots\right.$ $\left.\ldots, m_{i}, m_{i+1}-1, m_{i+2}, \ldots, m_{n},-m_{1}-1\right)$. Но длина такого веса превосходит длину старшего веса модуля $V_{m}$, следовательно, $X_{-n, i} q_{N}=0$. Наконец, $X_{0 n} q_{N}=0$, поскольку оператор $X_{0 n}=\left[X_{10}, X_{-n, 1}\right]$ является коммутатором двух других операторов, аннулируюших вектор $q_{N}$. Тем самым равенство $\mathfrak{b}_{n-1 / 2} q_{N}=\mathfrak{b}_{n} q_{N}$, а вместе с ним и равенство $\mathscr{D}_{N}=V_{m}$ доказаны.

Мы получили фильтрацию

$$
f=\left\{\mathscr{D}_{0}, \mathscr{D}_{1}, \ldots, \mathscr{D}_{N} ; \mathscr{D}_{i-1} \subset \mathscr{D}_{i}, i=1, \ldots, N, \mathscr{D}_{0}=0, \mathscr{D}_{N}=V_{m}\right\}
$$

$\left(\mathfrak{b}_{n-1 / 2}\right)$-подмодулей $V_{m}$. Фильтрация $f$ удовлетворяет условиям а), б) определения 3.3, что непосредственно вытекает из следующей формулы, описывающей спектры сужений факторов $f$ на $\mathfrak{b}_{n-1}$ :

$$
D_{i+1} /\left.D_{i}\right|_{\mathfrak{b}_{n-1}}=\bigoplus_{m_{1}-p_{1} \geqslant t_{1} \geqslant \cdots \geqslant m_{n-1}-p_{n-1} \geqslant t_{n-1} \geqslant m_{n}-\left[\frac{p_{n}}{2}\right]} V_{\left(t_{1}, \ldots, t_{n-1}\right)},
$$

если $q_{i+1}=z_{1,-n}^{p_{1}} \ldots z_{n-1,-n}^{p_{n-1}} z_{n 0}^{p_{n}}$.

Для доказательства формулы (6) нам потребуются некоторые приготовления. 
ОПРЕДЕЛЕНИЕ 3.4. Будем говорить, что тройка весов $t \in P_{n-1}^{B}, l \in P_{n}^{D}$, $m \in P_{n}^{B}$ образует кортеж $\{t, l, m\}$, если $t \rightarrow l \rightarrow m$.

Каждому кортежу $\{t, l, m\}$ сопоставим полустарший вектор $\omega(t, l, m) \in V_{m}^{+}$, определяемый формулой

$$
\begin{aligned}
\omega(t, l, m)= & z_{1 n}^{l_{1}-m_{2}} \ldots z_{n-1, n}^{l_{n-1}-m_{n}} z_{1,-n}^{m_{1}-l_{1}} z_{2,-n}^{t_{1}-l_{2}} \ldots z_{n-1,-n}^{t_{n-2}-l_{n-1}} \\
& \times z_{n 0}^{2 t_{n-1}-\left[\left|2 l_{n}+\frac{1}{2}\right|\right]} \Omega_{1}^{m_{2}-t_{1}} \Omega_{2}^{m_{3}-t_{2}} \ldots \Omega_{n-1}^{m_{n}-t_{n-1}}
\end{aligned}
$$

где $\Omega_{1}=\widehat{z_{1,-n} z_{2 n}}-z_{1 n} z_{2,-n}, \quad \Omega_{2}=\widehat{z_{2,-n z_{3 n}}}-z_{2 n} z_{3,-n}, \ldots, \quad \Omega_{n-2}=$ $z_{n-2,-n_{n} z_{n, n}}-z_{n-2, n} z_{n-1,-n}, \Omega_{n-1}=\widehat{z_{n-1,-n}}+\frac{1}{2} z_{n-1, n} z_{n 0}^{2}$, а символ означает, что данное слагаемое должно быть пропушено, если показатель степени $k$ в выражении $\Omega_{i}^{k}$ будет отрицательным. Далее, сопоставим каждому кортежу $\{t, l, m\}$ вектор $r(t, l, m)$, определяемый формулой

$$
r(t, l, m)=X_{n 1}^{l_{1}-t_{1}} \ldots X_{n, n-1}^{l_{n-1}-t_{n-1}} q_{l, m},
$$

где $q_{l, m}=z_{1,-n}^{m_{1}-l_{1}} z_{2,-n}^{m_{2}-l_{2}} \ldots z_{n-1,-n}^{m_{n-1}-l_{n-1}} z_{n 0}^{2 m_{n}-\left[\left|2 l_{n}+\frac{1}{2}\right|\right]}$.

ЗАмечАниЕ. Очевидно, что $2 m_{n}-\left[\left|2 l_{n}+\frac{1}{2}\right|\right] \geqslant 0$ даже для отрицательных значений $l_{n}$, так как $m_{n} \geqslant\left|l_{n}\right|$ ввиду $l \rightarrow m$.

Отношение линейного порядка, определенное вьше на множестве квазистарших векторов $\left\{q_{l, m}, l \in P_{n}^{D}\right\}$, мы перенесем на индексы этих векторов, принадлежашие $P_{n}^{D}$. А именно будем говорить, что $l \succ p\left(l, p \in P_{n}^{D}\right)$, если $q_{l, m}<q_{p, m}$. (Проверим, что отношение $\succ$ введено корректно. Условие $l \succ p$ равносильно тому, что $\left(l_{1}, \ldots, l_{n-1},\left[\left|2 l_{n}+\frac{1}{2}\right|\right]\right)>\left(p_{1}, \ldots, p_{n-1},\left[\left|2 p_{n}+\frac{1}{2}\right|\right]\right)$ лексикографически, поэтому не зависит от выбора $\mathfrak{b}_{n}$-модуля $V_{m}$.)

Лемма 3.1. а) Пусть $\{t, l, m\} u\{q, p, m\}-$ кортежи. Тогда если $t \neq q$, то

$$
\langle r(t, l, m), \omega(q, p, m)\rangle=0 .
$$

б) Пусть $\{t, l, m\} u\{t, p, m\}-$ кортежи. Тогда если $l \succ p$, mо

$$
\langle r(t, l, m), \omega(t, p, m)\rangle=0 .
$$

в) Для любого кортежа $\{t, l, m\}$

$$
\langle r(t, l, m), \omega(t, l, m)\rangle \neq 0 .
$$

ДокАЗАТЕЛЬСТво. а) Если $t \neq q$, то векторы $r(t, l, m)$ и $\omega(q, p, m)$ имеют разные веса относительно $\mathfrak{h}_{n}$ и потому ортогональны.

б) Имеем, пользуясь контравариантностью скалярного произведения $\langle$. , . $\rangle$,

$$
\langle r(t, l, m), \omega(t, p, m)\rangle=\left\langle q_{l, m}, X_{1 n}^{l_{1}-t_{1}} \ldots X_{n-1, n}^{l_{n-1}-t_{n-1}} \omega(t, p, m)\right\rangle .
$$

Явный вид операторов $X_{1 n}, \ldots, X_{n-1, n}$ был указан в $\S 2$. Максимальная степень по $z_{1 n}$ среди слагаемых, входяших в разложение $\omega(t, p, m)$, равна $p_{1}-t_{1}$, а 
минимальная степень дифференщирования по этомй переменной равна $l_{1}-t_{1}$. Мы видим, что такое дифференцирование, примененное к отдельному моному, будет отлично от нуля тогда и только тогда, когда $l_{1}=p_{1}$, множитель вида $z_{1,-n} z_{2 n}$ не входит в моном, а из операторов $X_{2 n}, \ldots, X_{n-1, n}$ не берутся дифференцирования по $z_{1 n}$. Пусть для некоторого монома $M$ все эти условия выполняются. Далее, максимальная степень $M$ по $z_{2 n}$ равна $p_{2}-t_{2}$. Вместе с тем минимальная степень дифференцирования по $z_{2 n}$, входящего в оператор $X_{1 n}^{l_{1}-t_{1}} \ldots X_{n-1, n}^{l_{n-1}-t_{n-1}}$, равна $l_{2}-t_{2}$. Таким образом, результат дифференцирования отличен от нуля тогда и только тогда, когда $l_{2}=p_{2}$, множитель вида $z_{2,-n} z_{3 n}$ не входит в моном $M$, а из операторов $X_{3 n}, \ldots, X_{n-1, n}$ не берутся дифференцирования по $z_{2 n}, \ldots$ и т.д.

Пусть $l_{1}=p_{1}, l_{2}=p_{2}, \ldots, l_{n-1}=p_{n-1}$. Тогда $l \succ p$ означает, что $\left[\left|2 l_{n}+\frac{1}{2}\right|\right]>\left[\left|2 p_{n}+\frac{1}{2}\right|\right]$. Тогда вес $q_{l, m}$ в базисе $\epsilon_{1}, \ldots, \epsilon_{n}$ таков:

$$
\left(l_{1}, \ldots, l_{n-1},-\sum_{i=1}^{n} m_{i}+\sum_{i=1}^{n-1} l_{i}+\left[\left|l_{n}+\frac{1}{2}\right|\right]\right)
$$

в то время как вес $X_{1 n}^{l_{1}-t_{1}} \ldots X_{n-1, n}^{l_{n-1}-t_{n-1}} \omega(t, p, m)$ в этом же базисе:

$$
\left(l_{1}, \ldots, l_{n-1},-\sum_{i=1}^{n} m_{i}+\sum_{i=1}^{n-1} l_{i}+\left[\left|2 p_{n}+\frac{1}{2}\right|\right]\right),
$$

и теперь ортогональность указанных векторов вытекает из того, что их веса относительно эрмитова оператора $X_{n n}$ различны.

в) Нам надо доказать, что

$$
\left\langle q_{l, m}, X_{1 n}^{l_{1}-t_{1}} \ldots X_{n-1, n}^{l_{n-1}-t_{n-1}} \omega(t, l, m)\right\rangle \neq 0 .
$$

Из доказательства б) видно, что единственное операторное слагаемое, входяшее в разложение $X_{1 n}^{l_{1}-t_{1}} \ldots X_{n-1, n}^{l_{n-1}-t_{n-1}}$ и даюшее ненулевой результат после дифференцирования, есть $\left(\frac{\partial}{\partial z_{1 n}}\right)^{l_{1}-t_{1}} \ldots\left(\frac{\partial}{\partial z_{n-1, n}}\right)^{l_{n-1}-t_{n-1}}$, а единственный моном, входящий в разложение $\omega(t, l, m)$, на котором это операторное слагаемое отлично от нуля, есть (с точностью до знака)

$$
z_{1 n}^{l_{1}-t_{1}} \ldots z_{n-1, n}^{l_{n-1}-t_{n-1}} z_{1,-n}^{m_{1}-l_{1}} \ldots z_{n-1,-n}^{m_{n-1}-l_{n-1}} z_{n 0}^{2 m_{n}-\left[\left|2 l_{n}+\frac{1}{2}\right|\right]}
$$

Имеем

$$
\begin{aligned}
& X_{1 n}^{l_{1}-t_{1}} \ldots X_{n-1, n}^{l_{n-1}-t_{n-1}} \omega(t, l, m)=\left(l_{1}-t_{1}\right) ! \ldots\left(l_{n-1}-t_{n-1}\right) ! \\
& \quad \times z_{1,-n}^{m_{1}-l_{1}} \ldots z_{n-1,-n}^{m_{n-1}-l_{n-1}} z_{n 0}^{2 m_{n}-\left[\left|2 l_{n}+\frac{1}{2}\right|\right]}=\prod_{i=1}^{n-1}\left(l_{i}-t_{i}\right) ! q_{l, m} .
\end{aligned}
$$

Тогда

$$
\langle r(t, l, m), \omega(t, l, m)\rangle=\prod_{i=1}^{n-1}\left(l_{i}-t_{i}\right) !\left|q_{l, m}\right|^{2} \neq 0 .
$$

Лемма полностью доказана. 
СЛЕДСТВИЕ 3.1. Система векторов $\{\omega(t, l, m) ;\{t, l, m\}-\kappa о р т е ж\}$ образует базис пространства $V_{m}^{+}$, состоящий из полустарших векторов.

ДокАЗАТЕЛЬСтво. Легко видеть, что вектор $\omega(t, l, m)$ - весовой вектор $V_{m}$, его вес в базисе $\epsilon_{1}, \ldots, \epsilon_{n}$ таков:

$$
\left(t_{1}, \ldots, t_{n-1},-\sum_{i=1}^{n} m_{i}+2 \sum_{i=1}^{n-1} l_{i}-\sum_{i=1}^{n-1} t_{i}+\left[\left|2 l_{n}+\frac{1}{2}\right|\right]\right) .
$$

Поскольку различные весовые подпространства $V_{m}$ линейно независимы, нам достаточно доказать линейную независимость векторов $\omega(t, l, m)$, имеющих один и тот же фиксированный вес. Пусть

$$
\alpha_{1} \omega\left(t, p_{1}, m\right)+\alpha_{2} \omega\left(t, p_{2}, m\right)+\cdots+\alpha_{s} \omega\left(t, p_{s}, m\right)=0,
$$

где веса $p_{1}, p_{2}, \ldots, p_{s}$ упорядочены так, что $p_{1} \succ p_{2} \succ \cdots \succ p_{s}$. Последовательно умножая это равенство скалярно на $r\left(t, p_{1}, m\right), r\left(t, p_{2}, m\right), \ldots, r\left(t, p_{s}, m\right)$, и применяя лемму 3.1 , получаем $\alpha_{1}=\alpha_{2}=\cdots=\alpha_{s}=0$.

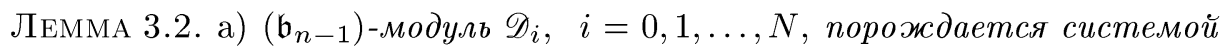
векторов $\left\{r(t, l, m) ;\{t, l, m\}-\right.$ кортеж, удовлетворяющий $\left.q_{l, m} \leqslant q_{i}\right\}$.

б) $\left(\mathfrak{b}_{n-1}\right)$-модуль $\mathscr{D}_{i}^{\perp}, \quad i=0,1, \ldots, N$, порождается системой векторов $\left\{\omega(t, l, m) ;\{t, l, m\}\right.$ - кортеж, удовлетворяющий $\left.q_{l, m}>q_{i}\right\}$.

ДокАЗАТЕЛЬСТво. Будем доказывать индукцией по $i$. При $i=0$ утверждение леммы вытекает из следствия 3.1 и предложения 2.2 . Пусть для $i=0,1, \ldots, k-1$ утверждение леммы доказано. Докажем его для $i=k$. Обозначим через $R$ $\left(\mathfrak{b}_{n-1}\right)$-подмодуль модуля $\mathscr{D}_{k}$, порожденный векторами $\{r(t, l, m) ;\{t, l, m\}$ - кортеж, удовлетворяющий $\left.q_{l, m} \leqslant q_{k}\right\}$. По предположению индукции $\left(\mathfrak{b}_{n-1}\right)$-модуль $\mathscr{D}_{k-1}$ порождается векторами $\left\{r(t, l, m) ;\{t, l, m\}\right.$ - кортеж, удовлетворяющий $q_{l, m}$ $\left.\leqslant q_{k-1}\right\}$. Поэтому $\mathscr{D}_{k-1} \subset R$. Тогда $R^{\perp} \subset \mathscr{D}_{k-1}^{\perp}$.

$\mathrm{C}$ другой стороны, по предположению индукции $\mathscr{D} \frac{\perp}{k-1}$ порождается системой векторов $S=\left\{\omega(t, l, m) ;\{t, l, m\}\right.$ - кортеж, удовлетворяющий $\left.q_{l, m}>q_{k-1}\right\}$. Пусть $r$ - произвольный полустарший вектор $\left(\mathfrak{b}_{n-1}\right)$-модуля $R^{\perp}$. Он представим в виде линейной комбинации векторов $S$ :

$$
r=\sum_{\substack{\{t, l, m\} \\ q_{l, m}=q_{k}}} \alpha_{(t, l, m)} \omega(t, l, m)+\sum_{\substack{\{t, l, m\} \\ q_{l, m}=q_{k+1}}} \beta_{(t, l, m)} \omega(t, l, m)+\ldots
$$

Умножая это равенство скалярно на $r(t, l, m)$, где $\{t, l, m\}$ - кортеж, удовлетворяющий $q_{l, m}=q_{k}$, и пользуясь леммой 3.1 , получаем $\alpha_{(t, l, m)}=0$ для всех $\{t, l, m\}$ таких, что $q_{l, m}=q_{k}$. Таким образом, $R^{\perp}$, как $\left(\mathfrak{b}_{n-1}\right)$-модуль, порождается системой векторов $S^{\prime}=\left\{\omega(t, l, m) ;\{t, l, m\}\right.$ - кортеж, удовлетворяющий $\left.q_{l, m}>q_{k}\right\}$.

Нам осталось доказать, что $R=\mathscr{D}_{k}$. Пусть $v$ - произвольньй ненулевой вектор $\mathscr{D}_{k}$. Покажем, что $v$ ортогонален системе векторов $S^{\prime}$. Если $v \in \mathscr{D}_{k-1}$, то $v$ ортогонален $S^{\prime}$ по предположению индукции. В общем случае $v=X q_{k}+v_{k-1}$, 
где $X \in U\left(\mathfrak{b}_{n-1 / 2}\right), v_{k-1} \in \mathscr{D}_{k-1}$. По теореме Пуанкаре-Биркгофа-Витта $X$ представим в виде

$$
X=\sum_{\substack{\alpha_{i} \geqslant 0, \beta_{i} \geqslant 0 \\ \gamma \geqslant 0}} c_{\alpha_{1}, \ldots, \alpha_{n-1}, \beta_{1}, \ldots, \beta_{n-1}, \gamma} X_{n 1}^{\alpha_{1}} \ldots X_{n, n-1}^{\alpha_{n-1}} X_{1,-n}^{\beta_{1}} \ldots X_{n-1,-n}^{\beta_{n-1}} X_{n 0}^{\gamma}
$$

где $c_{\alpha_{1}, \ldots, \alpha_{n-1}, \beta_{1}, \ldots, \beta_{n-1}, \gamma} \in U\left(\mathfrak{n}_{-}^{0}\right)$.

Теперь если $c_{\alpha_{1}, \ldots, \alpha_{n-1}, \beta_{1}, \ldots, \beta_{n-1}, \gamma}$ не содержит свободного члена, то для любого $\omega(t, l, m) \in S^{\prime}$

$$
\left\langle c_{\alpha_{1}, \ldots, \alpha_{n-1}, \beta_{1}, \ldots, \beta_{n-1}, \gamma} X_{n 1}^{\alpha_{1}} \ldots X_{n, n-1}^{\alpha_{n-1}} X_{1,-n}^{\beta_{1}} \ldots X_{n-1,-n}^{\beta_{n-1}} X_{n 0}^{\gamma} q_{k}, \omega(t, l, m)\right\rangle=0
$$

ввиду контравариантности скалярного произведения $\langle.$, . $\rangle$. Пусть теперь

$$
c_{\alpha_{1}, \ldots, \alpha_{n-1}, \beta_{1}, \ldots, \beta_{n-1}, \gamma}=c \in \mathbf{C}, \quad q_{k}=q_{p, m}
$$

и $\left(p_{1}, \ldots, p_{n-1},\left[\left|2 p_{n}+\frac{1}{2}\right|\right]\right)>\left(l_{1}, \ldots, l_{n-1},\left[\left|2 l_{n}+\frac{1}{2}\right|\right]\right)$ относительно лексикографического порядка. Покажем, что

$$
\left\langle X_{n 1}^{\alpha_{1}} \ldots X_{n, n-1}^{\alpha_{n-1}} X_{1,-n}^{\beta_{1}} \ldots X_{n-1,-n}^{\beta_{n-1}} X_{n 0}^{\gamma} q_{p, m}, \omega(t, l, m)\right\rangle=0 .
$$

Если эти векторы имеют различные веса относительно $\mathfrak{h}_{n}$, то они, очевидно, ортогональны. Предположим, что их веса совпадают. Они равны соответственно для левой и правой частей скалярного произведения (7):

$$
\begin{aligned}
\left(p_{1}+\beta_{1}-\alpha_{1}, \ldots, p_{n-1}\right. & +\beta_{n-1}-\alpha_{n-1} \\
& \left.-\sum_{i=1}^{n} m_{i}+\sum_{i=1}^{n-1} p_{i}+\left[\left|2 p_{n}+\frac{1}{2}\right|\right]+\sum_{i=1}^{n-1} \alpha_{i}+\sum_{i=1}^{n-1} \beta_{i}+\gamma\right)
\end{aligned}
$$

и

$$
\left(t_{1}, \ldots, t_{n-1},-\sum_{i=1}^{n} m_{i}-\sum_{i=1}^{n-1} t_{i}+2 \sum_{i=1}^{n-1} l_{i}+\left[\left|2 l_{n}+\frac{1}{2}\right|\right]\right) .
$$

Если $p_{1}>l_{1}$, то ввиду совпадения весов $\alpha_{1}=p_{1}-t_{1}+\beta_{1}>l_{1}-t_{1}$. Пользуясь контравариантностью $\langle.$, . $\rangle$, перепишем скалярное произведение $(7)$ :

$$
\left\langle X_{n 2}^{\alpha_{2}} \ldots X_{n, n-1}^{\alpha_{n-1}} X_{1,-n}^{\beta_{1}} \ldots X_{n-1,-n}^{\beta_{n-1}} X_{n 0}^{\gamma} q_{p, m}, X_{1 n}^{\alpha_{1}} \omega(t, l, m)\right\rangle .
$$

Но $X_{1 n}=\frac{\partial}{\partial z_{1 n}}$. Вместе с тем в произвольном слагаемом, входящем в разложение $\omega(t, l, m)$, степень по $z_{1 n}$ не превьшает $l_{1}-t_{1}$. Следовательно, $X_{1 n}^{\alpha_{1}} \omega(t, l, m)=0$. Рассуждая индуктивно, предположим, что $p_{1}=l_{1}, \ldots, p_{i-1}=l_{i-1}$, но $p_{i}>l_{i}$. Перепишем скалярное произведение (7) в виде

$$
\left\langle X_{n, i+1}^{\alpha_{i+1}} \ldots X_{n, n-1}^{\alpha_{n-1}} X_{1,-n}^{\beta_{1}} \ldots X_{n-1,-n}^{\beta_{n-1}} X_{n 0}^{\gamma} q_{p, m}, X_{i n}^{\alpha_{i}} \ldots X_{1 n}^{\alpha_{1}} \omega(t, l, m)\right\rangle .
$$


Ввиду совпадения весов, $\alpha_{1} \geqslant l_{1}-t_{1}, \ldots, \alpha_{i-1} \geqslant l_{i-1}-t_{i-1}, \alpha_{i}>l_{i}-t_{i}$. Повторяя рассуждение, проведенное при доказательстве леммы $3.1, б)$, получаем, что многочлен $X_{i-1, n}^{\alpha_{i-1}} \ldots X_{1 n}^{\alpha_{1}} \omega(t, l, m)$ отличен от нуля лишь при $\alpha_{1}=l_{1}-t_{1}, \ldots, \alpha_{i-1}=$ $l_{i-1}-t_{i-1}$. При этом всякий его ненулевой моном уже не содержит переменных $z_{1 n}, \ldots, z_{i-1, n}$. Поэтому в операторе $X_{i n}$ можно не рассматривать дифференцирования по $z_{1 n}, \ldots, z_{i-1, n}$. Тогда $X_{i n} \equiv \frac{\partial}{\partial z_{i n}}$ и $X_{i n}^{\alpha_{i}} \ldots X_{1 n}^{\alpha_{1}} \omega(t, l, m)=0$, что доказывает равенство (7).

Поскольку $\mathscr{D}_{k}$ ортогонален всем векторам системы $S^{\prime}$, то $S^{\prime} \in \mathscr{D}_{k}^{\perp}$. Ho $S^{\prime}$ порождает $\left(\mathfrak{b}_{n-1}\right)$-модуль $R^{\perp}$, откуда $R^{\perp} \subseteq \mathscr{D}_{k}^{\perp}$. Учитывая, что по построению $R \subseteq \mathscr{D}_{k}$, получаем $R=\mathscr{D}_{k}$. Лемма 3.2 доказана.

Обратимся теперь к доказательству равенств $(6) .\left(\mathfrak{b}_{n-1}\right)$-модули $\mathscr{D}_{i+1} / \mathscr{D}_{i}$ и $\mathscr{D}_{i} \perp \mathscr{D}_{i+1}^{\perp}$, очевидно, изоморфны. Но последний модуль согласно лемме 3.2 порождается полустаршими векторами $\left\{\omega(t, l, m)+\mathscr{D} \frac{\perp}{i+1} ;\{t, l, m\}\right.$-кортеж, удовлетворяющий $\left.q_{l, m}=q_{i+1}\right\}$. Вес вектора $\omega(t, l, m)+\mathscr{D}_{i+1}^{\perp}$ в базисе $\epsilon_{1}, \ldots, \epsilon_{n-1}$ пространства $\mathfrak{h}_{n-1}^{*}$ равен $t$, поэтому $\left(\mathfrak{b}_{n-1}\right)$-подмодуль модуля $D_{i}^{\perp} / D_{i+1}^{\perp}$, порождаемьй этим вектором, изоморфен $V_{\left(t_{1}, \ldots, t_{n-1}\right)}$. Условие $t \rightarrow l$, где $q_{l, m}=q_{i+1}=$ $z_{1,-n}^{p_{1}} \ldots z_{n-1,-n}^{p_{n-1}} z_{n 0}^{p_{n}}$, сводится к системе неравенств

$$
m_{1}-p_{1} \geqslant t_{1} \geqslant m_{2}-p_{2} \geqslant \cdots \geqslant m_{n-1}-p_{n-1} \geqslant t_{n-1} \geqslant m_{n}-\left[\frac{p_{n}}{2}\right]
$$

что доказывает равенства (6). Из этих равенств сразу следует, что построенная нами фильтрация $f$ удовлетворяет условию б) определения 3.3 .

Нам осталось доказать, что всякие два фактора $f$ либо не изоморфны, либо их прообразы в $V_{m}$ имеют разную степень нильпотентности относительно оператора $X_{n 0}$, который в используемой модели имеет вид

$X_{n 0}=\frac{\partial}{\partial z_{n 0}}+z_{1 n} \frac{\partial}{\partial z_{10}}+\cdots+z_{n-1, n} \frac{\partial}{\partial z_{n-1,0}}-z_{10} \frac{\partial}{\partial z_{1,-n}}-\cdots-z_{n-1,0} \frac{\partial}{\partial z_{n-1,-n}}$.

Пусть факторы $\mathscr{D}_{i+1} / \mathscr{D}_{i}$ и $\mathscr{D}_{j+1} / \mathscr{D}_{j}(i \neq j)$ порождаются соответственно образами квазистарших векторов

$$
z_{1,-n}^{p_{1}} \ldots z_{n-1,-n}^{p_{n-1}} z_{n 0}^{p_{n}} \quad \text { и } \quad z_{1,-n}^{l_{1}} \ldots z_{n-1,-n}^{l_{n-1}} z_{n 0}^{l_{n}}
$$

Если $p_{i}=l_{i}, i=1, \ldots, n-1$, a $\left[\frac{p_{n}}{2}\right] \neq\left[\frac{l_{n}}{2}\right]$, то эти факторы не изоморфны, поскольку (ввиду (6)) они не изоморфны как $\left(\mathfrak{b}_{n-1}\right)$-модули. Если же $\mathscr{D}_{i+1} / \mathscr{D}_{i}$ и $\mathscr{D}_{j+1} / \mathscr{D}_{j}$ как $\left(\mathfrak{b}_{n-1}\right)$-модули изоморфны, то $\left[\frac{p_{n}}{2}\right]=\left[\frac{l_{n}}{2}\right]$ и векторы $\left(p_{1}, \ldots, p_{n}\right)$ и $\left(l_{1}, \ldots, l_{n}\right)$ должны отличаться на вектор $(0, \ldots, 0,1)$. Такие факторы мы выше называли зеркально-сопряженными. Прообраз того фактора из пары зеркально-сопряженных факторов, у которого соответствуюший квазистарший вектор имеет большую степень по переменной $z_{n 0}$, имеет и большую (на 1 ) степень нильпотентности относительно оператора $X_{n 0}$. Таким образом, фильтрация $f$ удовлетворяет всем условиям определения 3.3 , и теорема 3.1 полностью доказана. 
ЗАмЕчАниЕ. Базис из полустарших векторов в пространстве $V_{m}^{+}$(см. следствие из леммы 3.1) легко может быть достроен до базиса во всем пространстве $V_{m}$. Для этого достаточно взять $\left(\mathfrak{b}_{n-1}\right)$-подмодули модуля $V_{m}$, порожденные базисными векторами $\omega(t, l, m),\{t, l, m$,$\} - кортеж. По предложению 2.2$ эти подмодули неприводимы и $V_{m}$ является их прямой суммой. Далее в каждом из этих $\left(\mathfrak{b}_{n-1}\right)$-подмодулей надо выбрать базис из полустарших (относительно $\mathfrak{b}_{n-2}$ ) векторов, и т.д. Построенный таким образом базис в пространстве $V_{m}$ является весовым (так как все векторы $\omega(t, l, m)$ весовые относительно $\left.\mathfrak{h}_{n}\right)$, хотя, вообще говоря, не является ортогональным.

\section{$\S 4$. Категория $\mathscr{L}$ конечномерных $\left(\mathfrak{b}_{n-1 / 2}\right)$-модулей}

В $\S 3$ мы получили большой класс конечномерных (не вполне приводимых, вообще говоря) ( $\left.\mathfrak{b}_{n-1 / 2}\right)$-модулей, которые возникают как факторы фильтраций $f$, разделяюших изоморфные компоненты $\left(\mathfrak{b}_{n-1}\right)$-модулей $V_{m}$ с помошью промежуточной подалгебры $\mathfrak{b}_{n-1 / 2}$. Ниже мы получим более полное описание этого класса в терминах модулей Верма и их конечномерных фактормодулей.

Пусть, по-прежнему, $P_{n}^{B}$ - множество старших весов $\mathfrak{b}_{n}$. Обозначим через $M$ категорию модулей Верма $M(\lambda)$ над $\mathfrak{b}_{n}$ со старшим весом $\lambda \in P_{n}^{B}$ (внимание: нередко модуль Верма $M(\lambda)$ обозначается $M(\lambda+\delta)$, где $\delta$-полусумма положительных корней $\mathfrak{b}_{n}$ (см., например, [10]); аналогичное замечание относится и к фактормодулю $L(\lambda))$ :

$$
M=\left\{M(\lambda) ; \lambda \in P_{n}^{B}\right\} .
$$

Напомним, что модуль Верма $M(\lambda)$ над $\mathfrak{b}_{n}$ определяется следующим образом:

$$
M(\lambda)=U\left(\mathfrak{b}_{n}\right) \bigotimes_{U\left(\mathfrak{b}^{+}\right)} \mathbf{C}_{\lambda}
$$

где алгебра Ли $\mathfrak{b}^{+}$действует на $\mathbf{C}_{\lambda}$ с помошью одномерного представления $\tau_{\lambda}$ : $\tau_{\lambda}(h+n)=\lambda(h)$ для любых $h \in \mathfrak{h}_{n}$ и $n \in \mathfrak{n}_{+}$. Пусть $v_{\lambda}=1 \otimes 1-$ старший вектор $M(\lambda)$. Тогда $X_{i i} v_{\lambda}=\lambda_{i} v_{\lambda}, i=1, \ldots, n$.

Обозначим через $\mathscr{M}(\lambda)$ циклический $\left(\mathfrak{b}_{n-1 / 2}\right)$-подмодуль модуля $M(\lambda)$, порожденный $v_{\lambda}$. Тогда наряду с категорией $M$ мы получаем категорию $\mathscr{M}$ модулей Верма над $\mathfrak{b}_{n-1 / 2}$ со старшим весом $\lambda$ :

$$
\mathscr{M}=\left\{\mathscr{M}(\lambda) ; \lambda \in P_{n}^{B}\right\} .
$$

Следуюшее предложение показывает, что модуль Верма $\mathscr{M}(\lambda), \lambda=\left(\lambda_{1}, \ldots, \lambda_{n}\right)$, фактически не зависит от последней компоненты $\lambda_{n}$ старшего веса $\lambda$.

ПрЕДЛОЖЕНИЕ 4.1. Пусть $\lambda, \mu \in \mathfrak{h}_{n}^{*} \cap P_{n}^{B}$ таковы, что их ограничения на $\mathfrak{h}_{n-1}$ совпадают. Тогда $\mathscr{M}(\lambda) \simeq \mathscr{M}(\mu)$.

Доказательство в точности повторяет доказательство предложения 4.1 в [4] и потому опушено.

Пусть $\{t, l, m\}$ - кортеж (в смысле определения 3.4), $t \in P_{n-1}^{B}, l \in P_{n}^{D}, m \in P_{n}^{B}$. Легко видеть, что полустарший вектор $\omega(t, l, m)$ является квазистаршим, если

$$
t_{1}=l_{1}, \quad t_{2}=l_{2}, \quad \ldots, \quad t_{n-1}=l_{n-1} .
$$


А именно

$$
\omega(l, l, m)=(-1)^{n-1} z_{1,-n}^{m_{1}-l_{1}} \ldots z_{n-1,-n}^{m_{n-1}-l_{n-1}} z_{n 0}^{2 m_{n}-\left[\left|2 l_{n}+\frac{1}{2}\right|\right]} .
$$

ОПРЕдЕЛЕниЕ 4.1. Каждому кортежу $\{l, l, m\}$ (т.е. кортежу, удовлетворяюшему (8)), сопоставим квазистарший вектор $q_{l, m}$, определяемьй равенством

$$
q_{l, m}=z_{1,-n}^{m_{1}-l_{1}} z_{2,-n}^{m_{2}-l_{2}} \ldots z_{n-1,-n}^{m_{n-1}-l_{n-1}} z_{n 0}^{2 m_{n}-\left[\left|2 l_{n}+\frac{1}{2}\right|\right]} .
$$

Следующее предложение суммирует доказанные в $\S 2,3$ утверждения о связи квазистарших векторов $q_{l, m}$ с факторами фильтраций $f$, разделяюших изоморфные компоненты $\left(\mathfrak{b}_{n-1}\right)$-модулей $\left.V_{m}\right|_{\mathfrak{b}_{n-1}}$.

ПРЕДЛОЖЕНИЕ 4.2. а) Система векторов $\left\{q_{l, m} ;\{l, l, m\}\right.$ - кортеж $\}$ образует ортогональныи базис пространства квазистариих векторов $V_{m}^{++}$.

б) Факторы фильтраций $f$, разделяющих изоморфные компоненты $\left(\mathfrak{b}_{n-1}\right)$ модулей $V_{m}$, являются ииклическими $\left(\mathfrak{b}_{n-1 / 2}\right)$-модулями, порожденными образами квазистариих векторов $q_{l, m}$.

в) Пусть $\mathscr{D}_{i+1} / \mathscr{D}_{i}-$ фактор фильтрации $f$, порожденный образом квазистаршего вектора $q_{l, m}$. Тогда его спектр как $\left(\mathfrak{b}_{n-1}\right)$-модуля таков:

$$
\mathscr{D}_{i+1} /\left.\mathscr{D}_{i}\right|_{\mathfrak{b}_{n-1}}=\bigoplus_{l_{1} \geqslant t_{1} \geqslant l_{2} \geqslant \cdots \geqslant l_{n-1} \geqslant t_{n-1} \geqslant\left|l_{n}\right|} V_{\left(t_{1}, \ldots, t_{n-1}\right)} .
$$

ДокАЗАТЕЛЬСТво. Часть а) является переформулировкой предложения 2.3. Часть б) вытекает из построения фильтраций $f$. Часть в) является следствием формулы (6).

Из предложения 4.2 вытекает, в частности, что $\left(\mathfrak{b}_{n-1 / 2}\right)$-факторы фильтраций $f$ как $\left(\mathfrak{b}_{n-1}\right)$-модули однозначно определяются первым индексом $l \in P_{n}^{D}$ соответствуюшего квазистаршего вектора $q_{l, m}$. При этом факторы, отвечающие зеркально-сопряженным весам $l=\left(l_{1}, \ldots, l_{n-1}, l_{n}\right) \in P_{n}^{D}$ и $l^{*}=\left(l_{1}, \ldots, l_{n-1},-l_{n}\right) \in P_{n}^{D}$, как $\left(\mathfrak{b}_{n-1}\right)$-модули изоморфны. Более того, ниже будет показано (теорема 4.2$)$, что эти факторы и как $\left(\mathfrak{b}_{n-1 / 2}\right)$-модули однозначно определяются индексом $l$ вектора $q_{l, m}$, т.е. их структура как $\left(\mathfrak{b}_{n-1 / 2}\right)$-модулей не зависит от того, из какого исходного $\mathfrak{b}_{n}$-модуля $V_{m}$ они были получены; а зеркально-сопряженные факторы, порожденные квазистаршими векторами $q_{l, m}$ и $q_{l, m}^{*}$, изоморфны и как $\left(\mathfrak{b}_{n-1 / 2}\right)$-модули. Этот факт будет сушественно использоваться ниже при определении категории $\mathscr{L}$ конечномерных $\left(\mathfrak{b}_{n-1 / 2}\right)$-модулей.

Хорошо известно, что модуль Верма $M(\lambda)$ обладает единственным наибольшим $\mathfrak{b}_{n}$-подмодулем $K$, отличным от $M(\lambda)$. При этом фактормодуль $M(\lambda) / K=L(\lambda)$ является (при $\lambda \in P_{n}^{B}$ ) конечномерным неприводимым $\mathfrak{b}_{n}$-модулем со старшим весом $\lambda$, т.е. $L(\lambda) \simeq V_{\lambda}$. Отображение $i: P_{n}^{B} \rightarrow \mathfrak{b}_{n}^{\wedge}$, сопоставляющие весу $\lambda \in P_{n}^{B}$ класс эквивалентности $[L(\lambda)]$ конечномерных неприводимых $\mathfrak{b}_{n}$-модулей, взаимно однозначно $[10$, теорема 7.2 .6$]$.

ОПРЕДЕЛЕНИЕ 4.2. Пусть $\mathscr{K}=\mathscr{M}(\lambda) \cap K$. Фактормодуль $\mathscr{M}(\lambda) / \mathscr{K}$ является, очевидно, конечномерным $\left(\mathfrak{b}_{n-1 / 2}\right)$-модулем со старшим весом $\lambda$, который мы будем обозначать через $\mathscr{L}(\lambda)$. 
ТЕОРЕМА 4.1. Пусть $v_{\lambda}$ (соответственно $\left.v_{\lambda}^{\prime}\right)$ - канонический образующий модуля $\mathscr{M}(\lambda)$ (соответственно $\mathscr{L}(\lambda))$. Пусть $I$ (соответственно $\left.I^{\prime}\right)$ - аннулятор элемента $v_{\lambda}$ (соответственно $\left.v_{\lambda}^{\prime}\right)$ в $U\left(\mathfrak{b}_{n-1 / 2}\right)$. Тогда:

а) $\quad I=U\left(\mathfrak{b}_{n-1 / 2}\right) \mathfrak{m}_{+}+\sum_{h \in \mathfrak{h}_{n-1}} U\left(\mathfrak{b}_{n-1 / 2}\right)(h-\lambda(h))$
б) $\quad I^{\prime}=I+U\left(\mathfrak{b}_{n-1 / 2}\right) X_{0, n-1}^{2 \lambda_{n-1}+1}+\sum_{j=1}^{n-1} U\left(\mathfrak{b}_{n-1 / 2}\right) X_{j+1, j}^{\lambda_{j}-\lambda_{j+1}+1}$.

Доказательство теоремы 4.1 имеет вычислительный характер и потому опущено. Отметим лишь, что утверждение а) теоремы легко следует из соответствующей теоремы для $\mathfrak{b}_{n}$-модуля $M(\lambda)$ [10, предложение 7.2.7] и предложения 4.1. Доказательство утверждения б) аналогично доказательству теоремы для симплектической серии, приведенному в заметке [11].

ТЕОРема 4.2. Пусть $f$ - фильтрация, разделяющая компоненты $\left(\mathfrak{b}_{n-1}\right)$-модуля $V_{m}$ (см. § 3). Пусть $\mathscr{D}_{i+1} / \mathscr{D}_{i}$ и $\mathscr{D}_{j+1} / \mathscr{D}_{j}-\oint$ бкторы фильтрачии $f$, порожденные образами квазистариих векторов $q_{\lambda, m} u q_{\lambda^{*}, m}, \lambda, \lambda^{*} \in P_{n}^{D}-$ зеркально-сопряженнье веса, $\{\lambda, \lambda, m\}-$ кортеж. Тогда

$$
\mathscr{D}_{i+1} / \mathscr{D}_{i} \simeq \mathscr{D}_{j+1} / \mathscr{D}_{j} \simeq \mathscr{L}\left(\lambda_{1}, \ldots, \lambda_{n-1},\left|\lambda_{n}\right|\right)
$$

ДокАЗАТЕЛЬСТво. При построении фильтрации $f$ линейный порядок во множестве квазистарших векторов был выбран таким образом, чтобы $\mathfrak{m}_{+}\left(q_{\lambda, m}\right) \subset \mathscr{D}_{i}$ и $\mathfrak{m}_{+}\left(q_{\lambda^{*}, m}\right) \subset \mathscr{D}_{j}$. Таким образом, любой элемент идеала $I$ аннулирует образ $q_{\lambda, m}$ в $\mathscr{D}_{i+1} / \mathscr{D}_{i}$, а также образ $q_{\lambda^{*}, m}$ в $\mathscr{D}_{j+1} / \mathscr{D}_{j}$. Тогда ввиду свойства универсальности индуцированных представлений существует единственный сюръективный $\left(\mathfrak{b}_{n-1 / 2}\right)$-гомоморфизм $\varphi: \mathscr{M}\left(\lambda_{1}, \ldots, \lambda_{n-1},\left|\lambda_{n}\right|\right) \rightarrow \mathscr{D}_{i+1} / \mathscr{D}_{i}$ такой, что $\varphi(1 \otimes 1)=q_{\lambda, m}+\mathscr{D}_{i}$. Аналогично, существует единственный сюръективный $\left(\mathfrak{b}_{n-1 / 2}\right)$-гомоморфизм $\psi: \mathscr{M}\left(\lambda_{1}, \ldots, \lambda_{n-1},\left|\lambda_{n}\right|\right) \rightarrow \mathscr{D}_{j+1} / \mathscr{D}_{j}$ такой, что $\psi(1 \otimes 1)=$ $q_{\lambda^{*}, m}+\mathscr{D}_{j}$. Далее, из спектрального разложения $\mathscr{D}_{i+1} / \mathscr{D}_{i}$ как $\left(\mathfrak{b}_{n-1}\right)$-модуля (предложение 4.2, в)) следует, что $X_{0, n-1}^{2 \lambda_{n-1}+1} q_{\lambda, m} \in \mathscr{D}_{i}, X_{k+1, k}^{\lambda_{k}-\lambda_{k+1}+1} q_{\lambda, m} \in \mathscr{D}_{i}$ $(k=1,2, \ldots, n-2)$ и $X_{n, n-1}^{\lambda_{n-1}-\left|\lambda_{n}\right|+1} q_{\lambda, m} \in \mathscr{D}_{i}$. Аналогично, поскольку спектральные характеристики $\left(\mathfrak{b}_{n-1}\right)$-модулей $\mathscr{D}_{i+1} / \mathscr{D}_{i}$ и $\mathscr{D}_{j+1} / \mathscr{D}_{j}$ совпадают, имеем $X_{0, n-1}^{2 \lambda_{n-1}+1} q_{\lambda^{*}, m} \in \mathscr{D}_{j}, \quad X_{k+1, k}^{\lambda_{k}-\lambda_{k+1}+1} q_{\lambda^{*}, m} \in \mathscr{D}_{j} \quad(k=1,2, \ldots, n-2)$ и $X_{n, n-1}^{\lambda_{n-1}-\left|\lambda_{n}\right|+1} q_{\lambda^{*}, m} \in \mathscr{D}_{j}$. Учитывая, что подмодуль $\mathscr{K}$ в $\mathscr{M}\left(\lambda_{1}, \ldots, \lambda_{n-1},\left|\lambda_{n}\right|\right)$ порождается согласно теореме 4.1 векторами $X_{0, n-1}^{2 \lambda_{n-1}+1}(1 \otimes 1), X_{k+1, k}^{\lambda_{k}-\lambda_{k+1}+1}(1 \otimes 1)$, $k=1, \ldots, n-2$, и $X_{n, n-1}^{\lambda_{n-1}-\left|\lambda_{n}\right|+1}(1 \otimes 1)$, получаем $\mathscr{K} \subseteq \operatorname{ker} \varphi$ и $\mathscr{K} \subseteq \operatorname{ker} \psi$. Тогда факторотображения $\tilde{\varphi}: \mathscr{L}\left(\lambda_{1}, \ldots, \lambda_{n-1},\left|\lambda_{n}\right|\right) \rightarrow \mathscr{D}_{i+1} / \mathscr{D}_{i}$ и $\tilde{\psi}: \mathscr{L}\left(\lambda_{1}, \ldots, \lambda_{n-1}\right.$, $\left.\left|\lambda_{n}\right|\right) \rightarrow \mathscr{D}_{j+1} / \mathscr{D}_{j}$ являются изоморфизмами $\left(\mathfrak{b}_{n-1 / 2}\right)$-модулей, поскольку как $\left(\mathfrak{b}_{n-1}\right)$-модули они изоморфны. 
ЗАмечАниЕ. Согласно способу построения фильтраций $f$, разделяющих изоморфные компоненты $\left(\mathfrak{b}_{n-1}\right)$-модулей $V_{m}$, факторы фильтраций $\mathscr{D}_{i+1} / \mathscr{D}_{i}$ и $\mathscr{D}_{j+1} / \mathscr{D}_{j}$, порожденные образами квазистарших векторов $q_{\lambda, m}$ и $q_{\lambda^{*}, m}$, обязаны иметь соседние номера, если $\lambda \neq \lambda^{*}$, т.е. $i$ и $j$ на самом деле удовлетворяют соотношению $|i-j|=1$. Однако при доказательстве теоремы 4.2 этот факт нами не использовался.

Обозначим через $\mathscr{L}$ категорию $\left(\mathfrak{b}_{n-1 / 2}\right)$-факторов фильтраций, разделяюших изоморфные компоненты $\left(\mathfrak{b}_{n-1}\right)$-модулей $V_{m}$ (морфизмами $\mathscr{L}$ являются гомоморфизмы $\left(\mathfrak{b}_{n-1 / 2}\right)$-модулей). Из теоремы 4.2 вытекает следущее простое следствие.

СлеДСТВИЕ. $\mathscr{L}=\left\{\mathscr{L}(\lambda) ; \lambda \in P_{n}^{B}\right\}$.

ДокАЗАТЕЛЬСТво. Пусть $i: \mathscr{L} \in P_{n}^{B}$ - отображение, сопоставляющее фактору, порожденному образом квазистаршего вектора $q_{\lambda, m}(\{\lambda, \lambda, m\}-$ кортеж, $\left.\lambda \in P_{n}^{D}, \quad m \in P_{n}^{B}\right)$, его "старший" вес $\left(\lambda_{1}, \ldots, \lambda_{n-1},\left|\lambda_{n}\right|\right)$. Но если $\lambda=$ $\left(\lambda_{1}, \ldots, \lambda_{n-1}, \lambda_{n}\right) \in P_{n}^{D}$, то $\left(\lambda_{1}, \ldots, \lambda_{n-1},\left|\lambda_{n}\right|\right) \in P_{n}^{B}$. Следовательно, отображение $i$ сюръективно (при этом $i$ сопоставляет зеркально-сопряженным факторам, порожденным $q_{\lambda, m}$ и $q_{\lambda^{*}, m}, \lambda \neq \lambda^{*}$, один и тот же вес $\left(\lambda_{1}, \ldots, \lambda_{n-1},\left|\lambda_{n}\right|\right)$ $\left.\in P_{n}^{B}\right)$. Далее, факторы $\mathscr{L}(\lambda)$ и $\mathscr{L}(\mu)$, соответствуюшие различным "старшим" весам $\lambda, \mu \in P_{n}^{B}, \lambda \neq \mu$, не изоморфны как $\left(\mathfrak{b}_{n-1 / 2}\right)$-модули ввиду того, что как $\left(\mathfrak{b}_{n-1}\right)$-модули они не изоморфны (предложение 4.2, в)).

Следующее предложение суммирует спектральные характеристики модулей $\mathscr{L}(\lambda)$.

ПРЕДЛОЖЕНИЕ 4.3. Для любого $\mathfrak{b}_{n}$-модуля $V_{m}, m \in P_{n}^{B}$,

$$
\left.V_{m}\right|_{\mathfrak{b}_{n-1 / 2}}=\underset{l \rightarrow m}{\dot{+}} \mathscr{L}(l),
$$

где $\dot{+}$ - полупрямая сумма (зачепление) $\left(\mathfrak{b}_{n-1 / 2}\right)$-модулей, берется по всем весам $l \in P_{n}^{D}$ таким, ито $l \rightarrow m$;

$$
\left.\mathscr{L}(l)\right|_{\mathfrak{b}_{n-1}}=\bigoplus_{t \rightarrow l} V_{t}
$$

Предложение 4.3 дает основание считать, что именно модули категории $\mathscr{L}$ отвечают за "промежуточные строчки" в таблищах Гельфанда-Цетлина, нумеруюших базисные векторы $\mathfrak{b}_{n}$-модулей $V_{m}$ (cp. (5)). В то же время категория $\mathscr{L}$ является не только категорией модулей над промежуточной между $\mathfrak{b}_{n}$ и $\mathfrak{b}_{n-1}$ алгеброй Ли $\mathfrak{b}_{n-1 / 2}$, но и модули этой категории (рассматриваемые с точностью до изоморфизма) находятся во взаимно однозначном соответствии со множеством старших весов $P_{n}^{B}$ (следствие из теоремы 4.2), что объясняет на первый взгляд неожиданное отнесение данной промежуточной алгебры Ли именно к серии $\mathfrak{b}$.

Итак, теперь мы можем различать изоморфные неприводимые компоненты $\left(\mathfrak{b}_{n-1}\right)$-модуля $V_{m}$, относя их к различньм (см. определение 3.3$)$ модулям категории $\mathscr{L}$. 
ПримеР. Рассмотрим сужение $\mathfrak{b}_{3}$-модуля $V=V_{(2,1,1)}$ на $\mathfrak{b}_{2}$. Согласно правилу ветвления $(5)$ в спектре получаемого $\mathfrak{b}_{2}$-модуля неприводимая компонента $V_{(1,1)}$ (изоморфная присоединенному представлению $\mathfrak{b}_{2}$ ) содержится шестикратно. Пользуясь конструкцией Желобенко [3], мы реализуем $\mathfrak{b}_{3}$-модуль $V$ в пространстве многочленов на максимальной унипотентной подгруппе $Z_{+} ; f(z)=$ $f\left(z_{12}, z_{13}, z_{10}, z_{1,-3}, z_{1,-2}, z_{23}, z_{20}, z_{2,-3}, z_{30}\right) \in V$ тогда и только тогда, когда $f(z)$ удовлетворяет системе дифференциальных уравнений

$$
\begin{gathered}
\left(\frac{\partial}{\partial z_{12}}+z_{23} \frac{\partial}{\partial z_{13}}+z_{20} \frac{\partial}{\partial z_{10}}+z_{2,-1} \frac{\partial}{\partial z_{1,-3}}-\left(\frac{z_{20}^{2}}{2}+z_{23} z_{2,-3}\right) \frac{\partial}{\partial z_{1,-2}}\right)^{2} f(z)=0 \\
\left(\frac{\partial}{\partial z_{23}}+z_{30} \frac{\partial}{\partial z_{20}}-\frac{z_{30}^{2}}{2} \frac{\partial}{\partial z_{2,-3}}\right) f(z)=0 \\
\left(\frac{\partial}{\partial z_{30}}\right)^{3} f(z)=0
\end{gathered}
$$

Система векторов $\left\{z_{1,-3}^{k} z_{30}^{s}, 0 \leqslant k \leqslant 1,0 \leqslant s \leqslant 2, k, s \in \mathbf{Z}\right\}$ образует ортогональный базис пространства $V_{(2,1,1)}^{++}$. Изоморфные друг другу неприводимые $\mathfrak{b}_{2}$-подмодули порождаются следуюшими шестью векторами:

1) $X_{31} v=z_{13}$,

2) $X_{31} X_{03} v=z_{13} z_{30}$,

3) $X_{31} X_{03}^{2} v=z_{13} z_{30}^{2}$,

4) $X_{-3,1} v=z_{1,-3}$,

5) $X_{-3,1} X_{03} v=z_{1,-3} z_{30}$

6) $X_{-3,1} X_{03}^{2} v=z_{1,-3} z_{30}^{2}$,

где $v$ - старший вектор $\mathfrak{b}_{3}$-модуля $V_{(2,1,1)}$.

Фильтрация $f\left(\mathfrak{b}_{3-1 / 2}\right)$-подмодулей $V$, разделяюшая изоморфные компоненты, состоит из шести ненулевых подмодулей: $\mathscr{D}_{1}, \mathscr{D}_{2}, \mathscr{D}_{3}, \mathscr{D}_{4}, \mathscr{D}_{5}, \mathscr{D}_{6}$, порожденных соответственно векторами $v=1, X_{03} v=z_{30}, X_{03}^{2} v=z_{30}^{2}, X_{-3,1} v=z_{1,-3}$, $X_{-3,1} X_{03} v=z_{1,-3} z_{30}, X_{-3,1} X_{03}^{2} v=z_{1,-3} z_{30}^{2}$. Легко видеть, что $X_{31} v$ имеет ненулевой образ в $\mathscr{D}_{1} / \mathscr{D}_{0} \simeq \mathscr{L}(2,1,1), X_{31} X_{03} v$ имеет ненулевой образ в $\mathscr{D}_{2} / \mathscr{D}_{1} \simeq$ $\mathscr{L}(2,1,-1), \quad X_{31} X_{03}^{2} v$ имеет ненулевой образ в $\mathscr{D}_{3} / \mathscr{D}_{2} \simeq \mathscr{L}(2,1,0), X_{-3,1} v-$ в $\mathscr{D}_{4} / \mathscr{D}_{3} \simeq \mathscr{L}(1,1,1), \quad X_{-3,1} X_{03} v-{ }_{\text {в }} \mathscr{D}_{5} / \mathscr{D}_{4} \simeq \mathscr{L}(1,1,-1)$, наконец $X_{-3,1} X_{03}^{2} v$ - в $\mathscr{D}_{6} / \mathscr{D}_{5} \simeq \mathscr{L}(1,1,0)$. Зеркально-сопряженные факторы $\mathscr{D}_{1} / \mathscr{D}_{0} \simeq \mathscr{L}(2,1,1)$ и $\mathscr{D}_{2} / \mathscr{D}_{1} \simeq \mathscr{L}(2,1,-1)$ хотя и изоморфны как $\left(\mathfrak{b}_{3-1 / 2}\right)$-модули, но их прообразы в $V$ различаются по степени нильпотентности оператора $X_{30}$. На $\mathscr{D}_{1}$ оператор $X_{30}$ тождественно равен нулю, а на прообразе $\mathscr{D}_{2} / \mathscr{D}_{1}$ оператор $X_{30}$ имеет степень нильпотентности 2: $X_{30} \neq 0$, но $X_{30}^{2}=0$.

Аналогичное замечание справедливо и для зеркально-сопряженных факторов $\mathscr{D}_{4} / \mathscr{D}_{3} \simeq \mathscr{L}(1,1,1)$ и $\mathscr{D}_{5} / \mathscr{D}_{4} \simeq \mathscr{L}(1,1,-1)$. На прообразе фактора $\mathscr{D}_{4} / \mathscr{D}_{3}$ оператор $X_{30}$ имеет степень нильпотентности 3 , а на прообразе $\mathscr{D}_{5} / \mathscr{D}_{4}$ - степень нильпотентности 4 . Таким образом, $\mathfrak{b}_{2}$-модуль $V_{(2,1,1)}$ допускает разделение изоморфных компонент с помощью промежуточной подалгебры $\mathfrak{b}_{3-1 / 2}$.

Автор глубоко признателен Д. П. Желобенко за полезные обсуждения. 


\section{Список литературы}

1. Вейль Г. Теория групп и квантовая механика. М.: Наука, 1986.

2. Гельфанд И. М., Цетлин М. Л. Конечномерные представления группы ортогональных матриц // ДАН СССР. 1950. Т. 71. №6. С. 1017-1020.

3. Желобенко Д. П. Классические группы. Спектральный анализ конечномерных представлений // УМН. 1962. Т. 17. № 1. С. 27-119.

4. Штепин В. В. Промежуточные алгебры Ли и их конечномерные представления // Изв. РАН. Сер. матем. 1993. Т. 57. №6. С. 176-198.

5. Штепин B. В. Разделение кратных точек спектра в редукции $\operatorname{sp}(2 n) \downarrow \operatorname{sp}(2 n-2)$ // Функцион. анализ и его прилож. 1986. Т. 20. № 4. С. 93-95.

6. Желобенко Д. П. Компактные группы Ли их представления. М.: Наука, 1970.

7. Jantzen J. G. Moduln mit einem höchsten Gewicht // Lect. Notes Math. 1979. B. 750. S. $1-182$.

8. Желобенко Д. П. Аналог базиса Гельфанда-Цетлина для симплектических алгебр Ли // УМН. 1987. Т. 42. №6. С. 193-194.

9. Серр Ж. П. Группы и алгебры Ли. М.: Мир, 1969.

10. Диксмье Ж.. Универсальные обертывающие алгебры. М.: Мир, 1978.

11. Штепин В. В. Об одном классе конечномерных $\operatorname{sp}(2 n-1)$-модулей // УМН. 1986. Т. 41. №3. C. 207-208.

Поступило в редакцию

19.VIII.1996 